\title{
CHARACTERIZATION OF PRIVILEGED POLYDOMAINS
}

\author{
BY
}

\author{
YUM-TONG SIU(1)
}

\begin{abstract}
This paper gives a number of equivalent conditions for a bounded polydomain to be privileged with respect to a coherent analytic sheaf in the sense of Douady. One of the equivalent conditions is in terms of the homological codimensions of the sheaf at the boundary of the polydomain. In the case of a polydisc, this condition about homological codimensions coincides with a conjecture of Douady. The other equivalent conditions concern some weaker concepts of privilegedness and the existence of privileged sets at the boundary.
\end{abstract}

0. In this paper we give a number of equivalent conditions for a bounded polydomain (with reasonable boundary) to be privileged with respect to a coherent analytic sheaf in the sense of Douady [1]. One of the equivalent conditions is in terms of the homological codimensions of the sheaf at the boundary of the polydomain. In the case of a polydisc, this condition about homological codimensions coincides with a conjecture of Douady. The other equivalent conditions concern some weaker concepts of privilegedness and the existence of privileged sets at the boundary.

To state the results, we have to introduce some notations and definitions. For a subset $E$ of a topological space, $E^{-}$and $\partial E$ denote respectively the topological closure and the topological boundary of $E .{ }_{n} \mathcal{C}$ denotes the sheaf of germs of holomorphic functions on $\mathbf{C}^{n}$.

(0.1) For a bounded open subset $G$ of $\mathbf{C}^{n}$, denote by $B(G)$ the set of all uniformly continuous holomorphic functions on $G . B(G)$ is a Banach space and is topologically isomorphic to the Banach space of all functions continuous on $G^{-}$and holomorphic on $G$.

(0.2) Suppose $\Omega$ is a Stein open subset of $C^{n}, G$ is a relatively compact open subset of $\Omega$, and $\mathcal{F}$ is a coherent analytic sheaf on $\Omega$. Suppose $\mathcal{F}$ admits a finite free resolution on $\Omega$, i.e. there exists on $\Omega$ an exact sequence of sheaf-

Received by the editors August 2, 1971.

AMS (MOS) subject classifications (1970). Primary 32C35; Secondary 46B99.

Key words and phrases. Coherent analytic sheaves, homological codimensions, privileged sets, Banach spaces.

(1) Part of the research for this paper was done while the author was supported by a grant from the National Science Foundation. 
homomorphisms of the form:

$$
0 \rightarrow \underset{n}{\Theta^{p} m} \rightarrow \cdots \rightarrow \Theta^{\vartheta^{p}} \rightarrow \Theta^{\Theta_{0}} \rightarrow \mathcal{T} \rightarrow 0
$$

Let

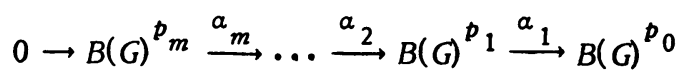

be induced by the preceding sequence. We introduce the following definitions of privilegedness.

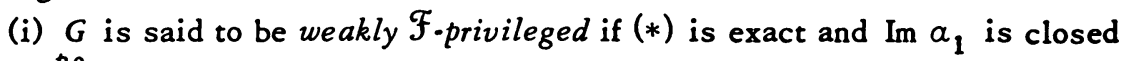
in $B(G)^{p_{0}}$.

(ii) $G$ is said to be $\mathcal{F}$-privileged if $(*)$ is direct-exact, i.e. $(*)$ is exact and, for $0 \leq i<m$, Im $\alpha_{i+1}$ is closed in $B(G)^{p_{i}}$ and there exists a closed subspace $E_{i}$ of $B(G)^{p_{i}}$ such that $B(G)^{p_{i}}=E_{i} \oplus \operatorname{Im} \alpha_{i+1}$.

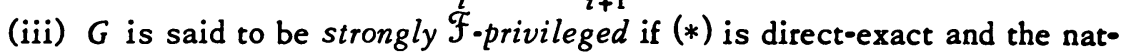
ural map Coker $\alpha_{1} \rightarrow \Gamma(G, \mathcal{F})$ is injective.

If $G$ is weakly $\mathcal{F}$-privileged, define $B(G, \mathcal{F})$ to be Coker $\alpha_{1}$.

These three definitions of privileged sets and the definition of $B(G, \mathcal{F})$ are independent of the choice of $\Omega$ and the choice of the finite free resolution of $\mathcal{F}$ on $\Omega$, because, by using Theorem B of Cartan-Oka, we can easily prove that any two finite free resolutions of $\mathcal{F}$ on a given Stein open subset of $\Omega$ become isomorphic finite free resolutions after we apply to each of them a finite number of modifications [4, Definition VI.F.1], i.e. after we apply to each of them a finite number of times the process of replacing it by its direct sum with some finite free resolution of the zero-sheaf which has only two nonzero terms (cf. [4, p. 202, VI.F.3]).

Note that $B\left(G,{ }_{n} \mathcal{C}^{p}\right)=B(G)^{p}$.

In the case where $G$ is a polydisc, the definition of $G$ being $\mathcal{F}$-privileged agrees with the definition given by Douady [1, p. 54, $\$ 7$, Definition 1$]$.

We introduce the following definitions of local privilegedness for bounded open subsets of $\mathbf{C}^{n}$ :

(iv) $G$ is said to be locally weakly $\mathcal{F}$-privileged if every point of $\partial G$ admits a basis $\mathfrak{U}$ of open neighborhoods in $\mathrm{C}^{n}$ such that $G \cap U$ is weakly $\mathcal{F}_{\text {-privileged }}$ for every $U \in \mathfrak{U}$.

(v) $G$ is said to be locally $\mathcal{F}$-privileged if every point of $\partial G$ admits a basis

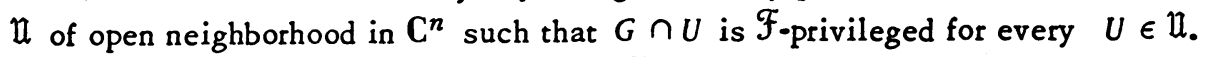

(vi) $G$ is said to be locally strongly $\mathcal{F}$-privileged if every point of $\partial G$ admits a basis $\mathfrak{U}$ of open neighborhood in $\mathbf{C}^{n}$ such that $G \cap U$ is strongly $\mathcal{F}_{\text {-priv- }}$ ileged. 
(vii) $G$ is said to be semilocally weakly $\mathcal{F}$-privileged if every point of $\partial G$ admits an open neighborhood $U$ in $\mathbf{C}^{n}$ such that $G \cap U$ is weakly $\mathcal{F}$-privileged.

(viii) $G$ is said to be semilocally $\mathcal{F}$-privileged if every point of $\partial G$ admits an open neighborhood $U$ in $\mathbf{C}^{n}$ such that $G \cap U$ is $\mathcal{F}$-privileged.

(ix) $G$ is said to be semilocally strongly $\mathcal{F}$-privileged if every point of $\partial G$ admits an open neighborhood $U$ in $\mathbf{C}^{n}$ such that $G \cap U$ is strongly $\mathcal{F}_{\text {-privileged. }}$

(0.3) A bounded open subset $G$ of $\mathbf{C}^{n}$ is called a polydomain if $G=G_{1} \times$ $\cdots \times G_{n}$ and each $G_{i}$ is a connected open subset of $\mathrm{C}(1 \leq i \leq n)$. For $0 \leq k$ $\leq n$, the boundary of order $k$ of $G$, denoted by $\partial_{k} G$, is defined as the set of all $\left(z_{1}, \ldots, z_{n}\right) \in G^{-}$such that $z_{i} \in \partial G_{i}$ for at least $k$ distinct values of $i$. Note that $\partial_{0} G=G^{-}, \partial_{1} G=\partial G$, and $\partial_{n} G$ is the distinguished boundary of $G$.

(0.4) For a bounded open subset $D$ of $\mathrm{C}, x \in \partial D$ is said to be a peak point of $B(D)$ if there exists $f \in B(D)$ such that the continuous extension $g$ of $f$ to $D^{-}$satisfies $g(x)=1$ and $|g(y)|<1$ for $y \in D^{-}-\{x\}$.

If $D$ equals the interior of $D^{-}$and there exists $d>0$ such that every component of $C-D^{-}$has diameter $\geq d$, then every point of $D$ is a peak point of $B(D)[3$, p. 205, VIII.4.4].

(0.5) For a coherent analytic sheaf $\mathcal{F}$ defined on an open subset $\Omega$ of $\mathbf{C}^{n}$, denote by $S_{k}(\mathcal{F})$ the set of all points $x \in \Omega$ such that the homological codimension of $\mathcal{F}_{x}$ over ${ }_{n} \mathcal{O}_{x}$ does not exceed $k . S_{k}(\mathcal{F})$ is always a subvariety of dimension $\leq k$ in $\Omega$ (see e.g. $[7$, p. 31, (1.11)]).

Now we are ready to state the results.

(0.6) Ma in Theorem. Suppose $\Omega$ is an open subset of $\mathbf{C}^{n}$ and $\mathcal{F}$ is a coberent analytic sheaf on $\Omega$ admitting a finite free resolution on $\Omega$. Suppose $G=G_{1} \times \cdots \times G_{n}$ is a polydomain in $\mathrm{C}^{n}$ such that $G \subset C \Omega$ and every point of $G_{i}$ is a peak point of $B\left(G_{i}\right)$ for $1 \leq i \leq n$. Then the following eleven statements are equivalent.

(i) $S_{k}(\mathcal{F}) \cap \partial_{k+1} G=\varnothing$ for $0 \leq k<n$.

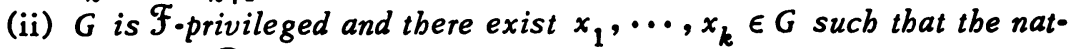
ural map $B(G, \mathcal{F}) \rightarrow \bigoplus_{i=1}^{k} \mathcal{F}_{x_{i}}$ is injective.

(iii) $G$ is strongly $\mathcal{F} \cdot$ privileged.

(iv) $G$ is $\mathcal{F}$-privileged.

(v) $G$ is weakly $\mathcal{F}$-privileged.

(vi) $G$ is locally strongly $\mathcal{F}$-privileged.

(vii) $G$ is locally $\mathcal{F}$-privileged.

(viii) $G$ is locally weakly $\mathcal{F}$-privileged.

(ix) $G$ is semilocally strongly $\mathcal{F}$-privileged.

(x) $G$ is semilocally $\mathcal{F}$-privileged.

(xi) $G$ is semilocally weakly $\mathcal{F}$-privileged. 
(0.7) Corollary. Suppose $P$ is a bounded open polydisc in $\mathbf{C}^{n}$ and $\mathcal{F}$ is a coberent analytic sheaf defined on some open neigbborbood of $P^{-}$. Then $P$ is F.privileged if and only if $S_{k}(\mathfrak{F}) \cap \partial_{k+1} P=\varnothing$ for $0 \leq k<n$.

The "if" part of Corollary (0.7) has also been proved by Pourcin [5]. Results similar to the "if" part of Corollary ( 0.7$)$ have been proved by Douady-FrischHirschowitz [2] for privileged sets which are defined by means of the Hilbert space of square-integrable holomorphic functions instead of the Banach space $B(G)$.

In this paper all topological spaces are assumed to be Hausdorff and have countable bases. $\mathbf{N}_{*}$ denotes the set of all nonnegative integers. Unless specified otherwise, the coordinates of $\mathbb{C}^{n}$ are denoted by $z_{1}, \ldots, z_{n^{*}}$ If $\mathfrak{U}=\left\{U_{i}\right\}$ is an open covering of a topological space $X$ and $\mathcal{F}$ is a sheaf on $X$ and $\xi \epsilon$ $C^{p}(\mathfrak{U}, \mathcal{F})$, then $\xi_{i_{0} \ldots i_{p}} \in \Gamma\left(U_{i_{0}} \cap \ldots \cap U_{i_{p}}, \mathcal{F}\right)$ denotes the value of $\xi$ at the simplex $\left(i_{0}, \cdots, i_{p}\right)$ of the nerve of $\mathfrak{U}$. A continuous linear map $f: E \rightarrow F$ of Fréchet spaces is called direct if $\operatorname{Im} f$ is closed in $F$ and there exist a closed subspace $E^{\prime}$ of $E$ and a closed subspace $F^{\prime}$ of $F$ such that $E^{\prime} \oplus \operatorname{Ker} f=E$ and $F^{\prime} \oplus \operatorname{Im} f=F$. A sequence of continuous linear maps of Fréchet spaces is called direct-exact if it is exact and if each map in it is direct.

1.

(1.1) Suppose $R$ is a local ring with maximal ideal $m$ and $M$ is a finitely generated $R$-module. A sequence $f_{1}, \ldots, f_{k} \in \mathrm{m}$ is called an $M$-sequence if $f_{i}$ is not a zero-divisor for $M / \sum_{j=1}^{i-1} f_{j} M$ for $1 \leq i \leq k$.

(1.2) Suppose $S$ and $\Omega$ are respectively open subsets of $C^{k}$ and $C^{n}$. Let $\pi: C^{k} \times C^{n} \rightarrow C^{k}$ be the natural projection. Suppose $\mathcal{F}$ is a coherent analytic sheaf on $S \times \Omega$. For $x \in S \times \Omega, \mathcal{F}$ is said to be $\pi$-flat at $x$ if $\mathcal{F}_{x}$ as a ${ }_{k} \mathcal{E}_{\pi(x)}$ module is flat over ${ }_{k} C_{\pi(x)}$.

Let $t_{1}, \cdots, t_{k}$ be the coordinates of $C^{k}$ and $\pi(x)=\left(t_{1}^{0}, \ldots, t_{k}^{0}\right)$. Then $\mathcal{F}$ is $\pi$-flat at $x$ if and only if $t_{1}-t_{1}^{0}, \cdots, t_{k}-t_{k}^{0}$ form an $\mathcal{F}_{x}$-sequence.

$\mathcal{F}$ is said to be $\pi \cdot f$ lat on a subset $E$ of $S \times \Omega$ if $\mathcal{F}$ is $\pi$-flat at every point of $E$.

(1.3) Lemma. Suppose $G$ is a bounded polydomain in $\mathrm{C}^{n}$ and $V$ is a sub. variety of some open neighborbood of $G^{-}$in $\mathrm{C}^{n}$. If $\operatorname{dim}_{x} V \geq k$ for some $x \in G^{-}$. then $V \cap \partial_{k} G \neq \varnothing$.

Proof. We can as sume without loss of generality (w.1.o.g.) that $V$ is puredimensional. We use induction on $k$. The case $k=0$ is trivial. Suppose $k>$ 0 and $V \cap \partial_{k} G=\varnothing$. Since by induction hypothesis $V \cap \partial_{k-1} G \neq \varnothing$, by reordering the coordinates of $C^{n}$, we can assume that there exists $z^{0}=\left(z_{1}^{0}, \cdots, z_{n}^{0}\right)$ 
$\epsilon G^{-} \quad V$ such that $z_{i}^{0} \in \partial G_{i}$ for $1 \leq i \leq k-1$. Let $z^{\prime}=\left(z_{1}^{0}, \ldots, z_{k-1}^{0}\right)$. It follows from $V \cap \partial_{k} G=\varnothing$ that

$$
V \cap\left(\left\{z^{\prime}\right\} \times \partial\left(G_{k+1} \times \cdots \times G_{n}\right)\right)=\varnothing .
$$

By [4, p. 106, III.B.17], for some open neighborhood $D$ of $\left(G_{k} \times \cdots \times G_{n}\right)^{-}$in $\mathrm{C}^{n-k+1}, \operatorname{dim} V \cap\left(\left\{z^{\prime}\right\} \times D\right) \leq 0$. Hence the dimension of the subvariety

$$
\left\{\left(z_{1}, \cdots, z_{n}\right) \in V \mid z_{i}=z_{i}^{0} \text { for } 1 \leq i \leq k-1\right\}
$$

at $z^{0}$ is 0 . This contradicts $\operatorname{dim}_{z^{0}} V \geq k$ [4, p. 115, III.C.14]. Q.E.D.

(1.4) Proposition. Suppose $G$ is a bounded polydomain in $\mathrm{C}^{n}$ and $\mathcal{F}$ is a coberent analytic sheaf defined on some open neighborbood of $G^{-}$. Then the following two conditions are equivalent.

(i) $S_{k}(\mathcal{F}) \cap \partial_{k+1} G=\varnothing$ for $0 \leq k<n$.

(ii) If $z^{0}=\left(z_{1}^{0}, \ldots, z_{n}^{0}\right) \in G^{-}$and $i_{1}, \ldots, i_{k}$ are distinct elements of $\{1$, $\cdots, n\}$ such that $z_{i}^{0} \in \partial G_{i}$ for $i=i_{1}, \ldots, i_{k}$, then $z_{i_{1}}-z_{i_{1}}^{0}, \cdots, z_{i_{k}}-z_{i_{k}}^{0}$ form an $\mathcal{F}_{z^{0}}$-sequence.

Proof. (ii) $\Rightarrow$ (i) is trivial. Suppose (i) holds. We use induction on $k$ to prove (ii). We can assume w.l.o.g. that $i_{\mu}=\mu$ for $1 \leq \mu \leq k$.

Consider first the case where $k=1$. Suppose $z_{1}-z_{1}^{0}$ is a zero-divisor for $\mathcal{F}_{z^{0}}$ By $[7$, p. $40,(1.18)]$, for some $p \geq 0$ there exists a $p$-dimensional branch $V$ of $S_{p}(\mathcal{F})$ such that $z^{0} \in V \subset\left\{z_{1}^{0}\right\} \times \mathrm{C}^{n-1}$. By applying Lemma (1.3) to the subvariety $V$ and the $(n-1)$-dimensional polydomain $\left\{z_{1}^{0}\right\} \times G_{2} \times \cdots \times G_{n}$, we conclude that $V$ intersects the boundary $Z$ of order $p$ of the $(n-1)$-dimensional polydomain $\left\{z_{1}^{0}\right\} \times G_{2} \times \cdots \times G_{n}$. This contradicts $S_{p}(\mathcal{F}) \cap \partial_{p+1} G=\varnothing$, because $p<n$ and $Z \subset \partial_{p+1} G$.

The case where $k>1$ is obtained by applying the induction hypothesis to the sheaf $\left(\mathcal{F}_{i}\left(z_{1}-z_{1}^{0}\right) \mathcal{F}\right) \mid\left\{z_{1}^{0}\right\} \times \mathbb{C}^{n-1}$ and the $(n-1)$-dimensional polydomain $\left\{z_{1}^{0}\right\} \times$ $G_{2} \times \cdots \times G_{n}$. Q.E.D.

2. In $\$ 2$ we gather together a couple of simple facts we need about holomorphic Banach bundles and their applications. These facts are treated in much greater generalities and details by Douady in [1].

(2.1) For Banach spaces $E_{0}, F_{0}$, we denote by $L\left(E_{0}, F_{0}\right)$ the Banach space of all continuous linear maps from $E_{0}$ to $F_{0}$.

Suppose $S$ is an open subset of $\mathbf{C}^{n}$ and $E$ is a holomorphic Banach bundle on $S$ with fiber $E_{0}$. For $s \in S$ we denote by $E_{s}$ the fiber of $E$ at $s$. For any open subset $U$ of $S$, we denote by $E \mid U$ the restriction of $E$ to $U$.

Suppose $G$ is a relatively compact open subset of $S$. A continuous section $f$ 
of $E$ over $G$ is said to be uniformly continuous if, for every open subset $U$ of $S$ for which there exists a trivialization $\alpha: E \mid U \cong U \times E_{0}$ and for every relatively compact open subset $W$ of $U$, the function $p a f \mid W$ is a uniformly continuous function from $W$ to $E_{0}$, where $p: U \times E_{0} \rightarrow E_{0}$ is the natural protection. A section of $E$ over $G$ is uniformly continuous if and only if it can be extended to a continuous section of $E$ over $G^{-}$. Denote by $B(G, E)$ the Banach space of all holomorphic sections of $E$ over $G$ which are uniformly continuous.

Suppose $F$ is a holomorphic Banach bundle on $S$ with fiber $F_{0}$. A map $\gamma$ : $E \rightarrow F$ is called a bundle-homomorphism if, for every open subset $U$ of $S$ for which there are trivializations $\alpha: E \mid U \cong U \times E_{0}$ and $\beta: F \mid U \cong U \times F_{0}$, there exists a holomorphic map $A(\cdot)$ from $U$ to $L\left(E_{0}, F_{0}\right)$ such that $\left(\beta \gamma \alpha^{-1}\right)(s, x)=$ $\left(s, A(s)_{x}\right)$ for $s \in U$ and $x \in E_{0}$. For any open subset $U$ of $S$, we denote by $\gamma \mid U$ the bundle-homomorphism $E|U \rightarrow F| U$ induced by $\gamma_{\text {. For }} s \in S$, we denote by $\gamma_{s}$ the map $E_{s} \rightarrow F_{s}$ induced by $\gamma$.

A bundle-homomorphism $\gamma: E \rightarrow F$ is called direct if both Ker $\gamma$ and Im $\gamma$ are holomorphic Banach bundles on $S$ and there exist holomorphic Banach bundles $E^{\prime}$ and $F^{\prime}$ on $S$ such that $E=E^{\prime} \oplus \operatorname{Ker} \gamma$ and $F=F^{\prime} \oplus \operatorname{Im} \gamma$. A sequence of bundle-homomorphisms is called direct-exact if it is exact and each bundle-homomorphism in it is direct.

(2.2) Suppose

$$
0 \rightarrow E^{(m)} \stackrel{\theta}{\rightarrow} E^{(m-1)} \rightarrow \ldots \rightarrow E^{(0)}
$$

is a complex of bundle-homomorphisms of holomorphic Banach bundles on $S$. If for some $s_{0} \in S$ the sequence

$$
0 \rightarrow E_{s_{0}}^{(m)} \stackrel{\theta_{s_{0}}}{\longrightarrow} E_{s_{0}}^{(m-1)} \rightarrow \ldots \rightarrow E_{s_{0}}^{(0)}
$$

is direct-exact, then there exists an open neighborhood $U$ of $s_{0}$ in $S$ such that the sequence

$$
0 \rightarrow E^{(m)}\left|U \rightarrow E^{(m-1)}\right| U \rightarrow \cdots \rightarrow E^{(0)} \mid U
$$

is direct-exact.

To prove this, it suffices to prove the case where $m=1$ and $E^{(1)}, E^{(0)}$ are both trivial bundles. Let $H$ be a closed subspace of $E_{s_{0}}^{(0)}$ which complements $\operatorname{Im} \theta_{s_{0}}$. Let $\sigma: E^{(1)} \oplus(S \times H) \rightarrow E^{(0)}$ be the bundlehomomorphism induced by $\theta$ and the inclusion map

$$
S \times H \hookrightarrow(S \times H) \oplus\left(S \times \operatorname{Im} \theta_{S_{0}}\right)=E^{(0)} .
$$


The existence of $U$ follows from the fact that, for some open neighborhood $U$ of $s_{0}$ in $S, \sigma \mid U$ is a bundle-isomorphism (i.e. there exists a bundle-homomorphism which is the inverse of $\sigma \mid U)$, which, in turn, follows from the fact that, if $P$ is a Banach space and $A(\cdot)$ is a holomorphic map from $S$ to $L(P, P)$ such that $A\left(s_{0}\right)$ admits an inverse $A\left(s_{0}\right)^{-1}$ in $L(P, P)$, then there exists an open neighborhood $U$ of $s_{0}$ in $S$ such that, for $s \in U, A(s)$ admits an inverse $A(s)^{-1}$ in $L(P, P)$ and the map $s \mapsto A(s)^{-1}$ is a holomorphic map from $U$ to $L(P, P)$.

(2.3) Suppose $S$.and $\Omega$ are respectively open subsets of $C^{k}$ and $C^{n}$, and $\mathcal{F}$ is a coherent analytic sheaf on $S \times \Omega$. For $s=\left(t_{1}^{0}, \cdots, t_{k}^{0}\right) \in S$, we denoce by $\mathcal{F}(s)$ the sheaf $\mathcal{F} / \Sigma_{i=1}^{k}\left(t_{i}-t_{i}^{0}\right) \mathcal{F}$, where $t_{1}, \cdots, t_{k}$ are the coordinates of $\mathbf{C}^{k}$. $\mathcal{F}(s)$ can be regarded in a natural way as a sheaf on $\Omega$.

For any positive integer $p$, we denote by $B\left(\Omega,{ }_{k+n} \Theta^{p}\right)$ the trivial Banach bundle on $S$ whose fiber is $B\left(\Omega,{ }_{n} \mathcal{C}^{p}\right)$.

(2.4) Suppose $S$ and $\Omega$ are respectively Stein open subsets of $C^{k}$ and $C^{n}$, and $\pi: S \times \Omega \rightarrow S$ is the natural projection. Suppose $\mathcal{F}$ is a $\pi$-flat coherent analytic sheaf on $S \times \Omega$ admitting a finite free resolution

$$
0 \rightarrow{ }_{k+n} \Theta^{p_{m}} \rightarrow \cdots \rightarrow_{k+n} \Theta^{p_{1}} \rightarrow_{k+n} \Theta^{p_{0}} \rightarrow \mathcal{F} \rightarrow 0
$$

on $S \times \Omega$. Suppose $s \in S$ and $G$ is a relatively compact open subset of $\Omega$ such that $G$ is $\mathcal{F}(s)$-privileged. Then there exists an open neighborhood $U$ of $s$ in $S$

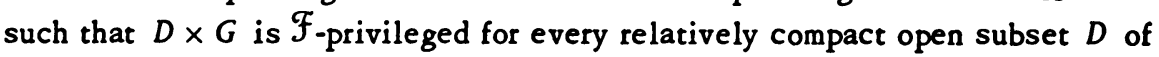
$U$. Moreover, if $G$ is strongly $\mathcal{F}(s)$-priviliged, then $U$ can be chosen so the $D \times$

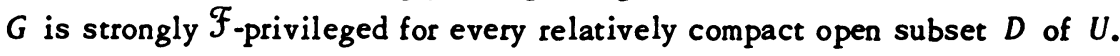

To prove this, consider the following sequence of bundle-homomorphisms induced by $(*)$ :

$$
0 \rightarrow B\left(G,{ }_{k+n} \Theta^{p}\right) \rightarrow \cdots \rightarrow B\left(G,{ }_{k+n} \Theta^{p}\right) \rightarrow B\left(G,{ }_{k+n} \Theta^{p} \%\right)
$$

Since $G$ is $\mathcal{F}(s)$-privileged and since by the $\pi$-flatness of $\mathcal{F}$ the sequence

$$
0 \rightarrow{ }_{k+n} \Theta^{p} m(s) \rightarrow \cdots \rightarrow_{k+n} \mathcal{O}^{p}(s) \rightarrow_{k+n} \Theta^{p} 0(s) \rightarrow \mathcal{F}(s) \rightarrow 0
$$

induced by $(*)$ is exact, we conclude that the sequence (\#), when restricted to the singleton $\{s\}$, is direct-exact. By $(2.2)$ there exists an open neighborhood $U$ of $s$ in $S$ such that (\#) is direct-exact on $U$. Our assertion follows from the fact that $B\left(D, B\left(G,{ }_{k+n} \mathcal{C}^{p_{i}}\right)\right)$ is topologically isomorphic to $B\left(D \times G,{ }_{k+n} \mathcal{O}^{p_{i}}\right)$ for any relatively compact open subset $D$ of $U(0 \leq i \leq m)$.

3.

(3.1) Suppose $X$ is a topological space. A sheaf $\mathcal{F}$ of $\mathrm{C}$-vector spaces on $X$ is called a Fréchet sheaf if the following two conditions are satisfied.

(i) For any open subset $W$ of $X, \Gamma(W, \mathcal{F})$ carries a Fréchet space structure. 
(ii) For open subsets $W^{\prime} \subset W$ of $X$ the restriction map $\Gamma(w, \mathcal{F}) \rightarrow \Gamma\left(W^{\prime}, \mathcal{F}\right)$ is continuous.

If $\mathfrak{U}$ is a countable open covering of $X$, then $C^{p}(\mathfrak{U}, \mathcal{F})$ has a natural Fréchet space structure.

If $\mathcal{G}$ is another Fréchet sheaf on $X$, then a sheaf-homomorphism $\phi: \mathcal{G} \rightarrow \mathcal{F}$ is called a Frécbet-sheaf-homomorphis $m$ if for every open subset $W$ of $X$ the map $\Gamma(w, \mathcal{G}) \rightarrow \Gamma(w, \mathcal{F})$ induced by $\phi$ is continuous.

The kernel of a Fréchet-sheaf-homomorphism is a Fréchet sheaf.

(3.2) Suppose $X$ is a topological space. A collection $\mathcal{E}$ of open subsets of $X$ is said to be a superbasis for the topology of $X$ if

(i) $\mathcal{E}$ is a basis for the topology of $X$, and

(ii) for any $E \in \mathcal{E}$ and any compact subset $A$ of $E$ there exists $E^{\prime} \in \mathcal{E}$ such that $A \subset E^{\prime} \subset C E$.

A Fréchet sheaf $\mathcal{F}$ on $X$ is said to be constructively fine relative to $\mathcal{E}$ if for every pair $E^{\prime} \subset C E$ of members of $\mathcal{E}$ and every finite covering $\mathfrak{U}$ of $E$ by members of $\mathcal{E}$ there exists a finite covering $\mathfrak{U}^{\prime}$ of $E^{\prime}$ by members of $\mathcal{E}$ which refines $\mathfrak{U}$ and there exists for $p \geq 1$ a continuous linear map

$$
a: Z^{p}(\mathfrak{U}, \mathcal{F}) \rightarrow C^{p-1}\left(\mathfrak{U}^{\prime}, \mathcal{F}\right)
$$

such that $\delta a$ is the map $Z^{p}(\mathfrak{U}, \mathcal{F}) \rightarrow Z^{p}\left(\mathfrak{U} \mathfrak{U}^{\prime}, \mathcal{F}\right)$ induced by the refinement map $\mathfrak{U}^{\prime} \rightarrow \mathfrak{U}$, where $\delta: C^{p-1}\left(\mathfrak{U}^{\prime}, \mathcal{F}\right) \rightarrow Z^{p}\left(\mathfrak{U}^{\prime}, \mathcal{F}\right)$ is the coboundary map.

(3.3) Suppose $X$ is a topological space and $\mathscr{E}$ is a superbasis for the topology of $X$. Suppose

$$
0 \rightarrow \stackrel{\odot}{*}_{n} \stackrel{\phi^{(n)}}{\longrightarrow} \ldots \stackrel{\phi^{(2)}}{\longrightarrow} \stackrel{\ominus}{\longleftarrow}_{1} \stackrel{\phi^{(1)}}{\longrightarrow} \stackrel{\ominus}{0}_{0}
$$

is an exact sequence of Fréchet-sheaf-homomorphisms on $X$.

Introduce the following notations: For every pair $E^{\prime} \subset C E$ of members of $\mathcal{E}$, denote by $\phi_{E}^{(\mu)}$ the map $\Gamma\left(E, \varrho_{\mu}\right) \rightarrow \Gamma\left(E, \varrho_{\mu-1}\right)$ induced by $\phi^{(\mu)}$, and denote by $\rho_{E^{\prime}, E}^{(\mu)}$ the restriction map $\Gamma\left(E, \varrho_{\mu}\right) \rightarrow \Gamma\left(E^{\prime}, \varrho_{\mu}\right)$.

The sequence $(*)$ is said to be locally direct relative to $\mathcal{E}$ if for $x \in E \in \mathcal{E}$ there exists. $E^{\prime} \in \mathcal{E}$ with $x \in E^{\prime} \subset E$ satisfying the following condition:

There exists a continuous linear map $\alpha_{E^{\prime}, E}^{(\mu)}: \Gamma\left(E, \stackrel{\rho}{+}_{\mu}\right) \rightarrow \Gamma\left(E^{\prime}, e_{\mu+1}^{e}\right)$

for $0 \leq \mu<n$ such that $\phi_{E^{\prime}}^{(\mu+1)} \alpha_{E^{\prime}, E}^{(\mu)} \phi_{E}^{(\mu+1)}=\rho_{E^{\prime}, E}^{(\mu)} \phi_{E}^{(\mu+1)}$.

The sequence $(*)$ is said to be globally direct relative to $\mathcal{E}$ if for every pair $E^{\prime} i C E$ of members of $\mathcal{E}$ the condition (\#) holds 
(3.4) Proposition. Suppose $X$ is a topological space and

$$
0 \rightarrow \mathscr{\complement}_{n} \stackrel{\phi^{(n)}}{\longrightarrow} \ldots \stackrel{\phi^{(2)}}{\longrightarrow} \mathscr{\complement}_{1} \stackrel{\phi^{(1)}}{\longrightarrow} \stackrel{\bigodot}{0}_{0}
$$

is an exact sequence of Fréchet-sheaf-bomomorphisms on $X$. Suppose $\mathcal{E}$ is a superbasis for the topology of $X$ and $\mathfrak{Q}_{\mu}$ is constructively fine relative to $\mathscr{G}$ for $0 \leq \mu \leq n$. If the sequence is locally direct relative to $\mathcal{E}_{\text {, then }}$

(i) the sequence is globally direct relative to $\mathcal{E}$ and

(ii) for every pair $E^{\prime} \subset C E$ of members of $\mathcal{E}$ the image of the map $\Gamma\left(E^{\prime}, \mathfrak{\aleph}_{1}\right)$ $\rightarrow \Gamma\left(E^{\prime}, \mathfrak{Q}_{0}\right)$ induced by $\phi^{(1)}$ contains the image of the restriction map $\Gamma\left(E, \operatorname{Im} \phi^{(1)}\right) \rightarrow \Gamma\left(E^{\prime}, \operatorname{Im} \phi^{(1)}\right)$.

Proof. By adding a zero term to the left of the sequence, we can assume w.1.o.g. that $\mathscr{\complement}_{n}=0$. In this proof we use the notations of (3.3) and the following notations: The letter $E$ (with or without primes) denotes always an element of $\mathcal{E}$. $\alpha_{E^{\prime}, E}^{(\mu)}$ always denotes a map satisfying (\#) of (3.3). For a collection $\mathfrak{U}$ of open subsets of $X$ and a sheaf $\mathcal{F}$ on $X, \delta^{p}(\mathfrak{U}, \mathcal{F})$ denotes the coboundary map $C^{p}(\mathfrak{U}, \mathfrak{F}) \rightarrow C^{p+1}(\mathfrak{U}, \mathfrak{F})$. If $\mathfrak{U}^{\prime}$ is another collection of open subsets of $X$ which refines $\mathfrak{U}$ by means of an index map $\tau$, then $r^{p}\left(\mathfrak{U}^{\prime}, \mathfrak{U}, \mathfrak{F}\right)$ denotes the map $Z^{p}(\mathfrak{U}, \mathcal{F}) \rightarrow Z^{p}\left(\mathfrak{U}^{\prime}, \mathcal{F}\right)$ induced by $\tau$.

Let $\varrho_{\mu}=\operatorname{Im} \phi^{(\mu+1)}(0 \leq \mu<n)$ and, for $E^{\prime} \subset E$, let $r_{E^{\prime}, E}^{(\mu)}$ denote the restriction map $\Gamma\left(E, \varrho_{\mu}\right) \rightarrow \Gamma\left(E^{\prime}, \varrho_{\mu}\right)$.

Since $\mathscr{\complement}_{\mu}$ is constructively fine relative to $\mathcal{E}$, the restriction map $H^{\nu}\left(E, \mathscr{\varrho}_{\mu}\right)$ $\rightarrow H^{\nu}\left(E^{\prime}, \varrho_{\mu}\right)$ is 0 for $E^{\prime} \subset C E, \nu \geq 1$, and $0 \leq \mu \leq n$. From this we conclude that

$$
\operatorname{Im} r_{E^{\prime}, E}^{(\mu)} \subset \operatorname{Im} \phi_{E^{\prime}}^{(\mu+1)} \text { for } E^{\prime} \subset \subset E \text { and } 0 \leq \mu<n \text {. }
$$

Conclusion (ii) of the proposition is proved.

We are going to prove that $\varrho_{0}$ is a Fréchet sheaf by showing that $\Gamma\left(E, \varrho_{0}\right)$ is closed in $\Gamma\left(E, \varrho_{0}\right)$ for all $E$. Suppose $f \in \Gamma\left(E, \mathfrak{\complement}_{0}\right)$ belongs to the topological closure of $\Gamma\left(E, \varrho_{0}\right)$. For $x \in E$ we can find $x \subset E^{\prime} \subset E^{\prime \prime} \subset \subset E$ such that $\alpha_{E^{\prime}}^{(0)}$, $E^{\prime \prime}$ exists. By $(A), \phi_{E^{\prime}}^{(1)}$ maps $\alpha_{E^{\prime}, E^{\prime \prime}}^{(0)} \rho_{E^{n}, E^{\prime}}^{(0)}$ to $\rho_{E^{\prime}, E^{\prime}}^{(0)}$. Hence $f_{x} \in\left(\varrho_{0}\right)_{x^{\prime}} \cdot \varrho_{0}$ is a Fréchet sheaf.

We are going to prove the following:

For $x \in E$ there exist $x \in E^{\prime} \subset E$ and a continuous linear map

(B) $\quad \beta_{E^{\prime}, E}^{(\mu)}: \Gamma\left(E, \stackrel{\odot}{\mathscr{J}}_{\mu}\right) \rightarrow \Gamma\left(E^{\prime}, \stackrel{P}{\mu+1}\right)$ for $0 \leq \mu<n$ such that $\phi_{E^{\prime}}^{(\mu+1)} \beta_{E^{\prime}, E}^{(\mu)}=r_{E^{\prime}, E}^{(\mu)}$. 
We can find $x \in E^{\prime} \subset E^{\prime \prime} \subset \subset E$ such that $\alpha_{E^{\prime}, E}^{(\mu)}$ exists. By $(A), \beta_{E^{\prime}, E}^{(\mu)}=$

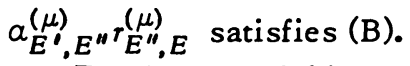

For the rest of this proof, $\beta_{E^{\prime}, E}^{(\mu)}$ denotes always a map satisfying (B).

We are going to prove the following two satements for $0 \leq \mu<n$ by descending induction on $\mu$ :

$\beta_{E^{\prime}, E}^{(\mu)}$ exists for $E^{\prime} \subset \subset E$.

(D) ${ }_{\mu}$

$\varrho_{\mu}$ is constructively fine relative to $\tilde{E}$.

Since $\mathscr{Q}_{n}=0,(C)_{n-1}$ and $(D)_{n-1}$ are trivial. Assume that $\mu<n-1$ and that $(C)_{\mu+1}$ and

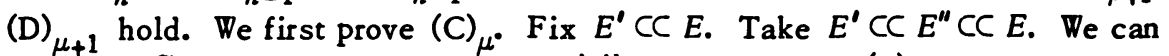
find $U_{i} \in \mathcal{E}, 1 \leq i \leq k$, such that $E^{\prime \prime} \subset \bigcup_{i=1}^{k} U_{i} \subset E$ and $\beta_{U_{i}, E}^{(\mu)}$ exists. By replacing $U_{i}$ by $U_{i} \cap E^{N}$ and replacing $\beta_{U_{i}, E}^{(\mu)}$ by $\rho_{U_{i} \cap E^{N}, U_{i}}^{(\mu+1)} \beta_{U_{i}, E}^{(\mu)}$, we can assume that $E^{\prime \prime}=\bigcup_{i=1}^{k} U_{i}$. Let

$$
\mathfrak{U}=\left\{U_{i}\right\}_{i=1}^{k} \text { and } \beta_{i}=\beta_{U_{i}}^{(\mu)}, E^{\bullet}
$$

Define

$$
\xi: \Gamma\left(E, \varrho_{\mu}\right) \rightarrow Z^{1}\left(\mathfrak{H}, \varrho_{\mu+1}\right)
$$

by

$$
\xi(f)_{i j}=\left(\beta_{j}(f)-\beta_{i}(f)\right) \mid U_{i} \cap U_{j}
$$

for $1 \leq i, j \leq k . \quad B y(D)_{\mu+1}$ there exists a finite covering $\mathfrak{U}^{\prime}=\left\{U_{i}^{\prime}\right\}_{i=1}^{l}$ of $E^{\prime}$ by members of $\mathcal{E}$ which refines $\mathfrak{U}$ by means of an index map $r:\{1, \ldots, l\} \rightarrow$ $\{1, \cdots, k\}$ and there exists a continuous linear map $\eta: Z^{1}\left(\mathfrak{U}, \varrho_{\mu_{+1}}\right) \rightarrow$ $C^{0}\left(\mathfrak{U}^{\prime}, \varrho_{\mu+1}\right)$ such that $\delta^{0}\left(\mathfrak{U}^{\prime}, \varrho_{\mu+1}\right)^{\prime}=\tau^{1}\left(\mathfrak{U}^{\prime}, \mathfrak{u}, \varrho_{u+1}\right)$. The map $\beta_{E}^{(\mu)}, E$ defined by

$$
\beta_{E^{\prime}, E}^{(\mu)}(f) \mid U_{i}^{\prime}=\left(\beta_{\tau(i)}(f) \mid U_{i}^{\prime}\right)-\eta \xi(f)_{i}
$$

for $1 \leq i \leq l$ satisfies the requirement. $(C)_{\mu}$ is proved.

To prove (D) ${ }_{\mu}$, fix $E^{\prime} \subset C E$ and a finite covering $\mathfrak{U}=\left\{U_{i}\right\}_{i=1}^{k}$ of $E$ by mem-

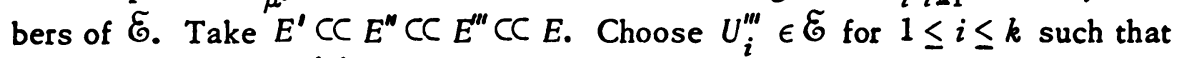
$U_{i}^{\prime \prime \prime} \subset \subset U_{i}$ and $E^{\prime \prime \prime}=\bigcup_{i=1}^{k} U_{i}^{\prime \prime \prime}$. Let $\mathfrak{U}^{\prime \prime \prime}=\left\{U_{i}^{\prime \prime \prime}\right\}_{i=1}^{k}$. By $(D)_{\mu_{+1}}$ there exists a finite covering $\mathfrak{U}^{\prime \prime}$ of $E^{\prime \prime}$ by members of $\mathcal{E}$ such that $\mathfrak{U}^{\prime \prime}$ refines $\mathfrak{U}^{\prime \prime \prime}$ by means of an index map $\tau_{1}$ and such that for $p \geq 1$ there exists a continuous linear map $\gamma_{p}$ :

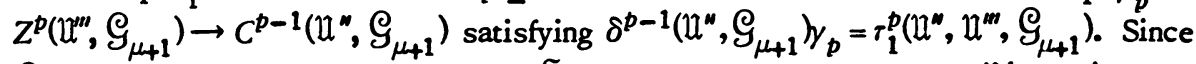
$\mathfrak{S}_{\mu+1}$ is constructively fine relative to $\mathcal{E}$, there exists a finite covering $\mathfrak{U}^{\prime}$ of $E^{\prime}$ by members of $\mathcal{E}$ such that $\mathfrak{U}$ 'refines $\mathfrak{U}^{\prime \prime}$ by means of an index map $r_{2}$ and such that for $p \geq 1$ there exists a continuous linear map 


$$
\nu_{p}: Z^{p}\left(\mathfrak{U}^{\prime \prime}, \mathfrak{\complement}_{\mu+1}\right) \rightarrow C^{p-1}\left(\mathfrak{U}^{\prime}, \stackrel{\complement}{\perp}_{\mu+1}\right)
$$

satisfying

$$
\delta^{p-1}\left(\mathfrak{U}^{\prime}, \mathfrak{\complement}_{\mu+1}\right) \nu_{p}=\tau_{2}^{p}\left(\mathfrak{U}^{\prime}, \mathfrak{U}^{\prime \prime}, \stackrel{\bigodot}{\perp}_{\mu+1}\right) .
$$

Fix $p \geq 1$. By $(\mathrm{C})_{\mu}$, for $1 \leq i_{0}<\cdots<i_{p} \leq k$ there exists

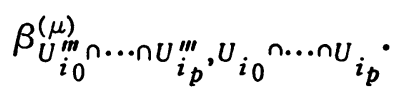

The collection of these maps defines a continuous linear map $\beta: Z^{p}\left(\mathfrak{U}, \varrho_{\mu}\right) \rightarrow$ $C^{p}\left(\mathfrak{U l}^{n}, \mathfrak{\complement}_{\mu+1}\right)$.

Let $\stackrel{\psi}{\psi}: C^{p-1}\left(\mathfrak{u}^{\prime}, \varrho_{\mu+1}\right) \rightarrow C^{p-1}\left(\mathfrak{u}^{\prime}, \varrho_{\mu}\right)$ be induced by $\phi^{(\mu+1)}$. Define $\theta$ : $Z^{p}\left(\mathfrak{U}, \varrho_{\mu}\right) \rightarrow C^{p-1}\left(\mathfrak{U}^{\prime}, \varrho_{\mu}\right)$ by

$$
\theta=\psi \nu_{p}\left(\tau^{p}\left(\mathfrak{u}^{\prime}, \mathfrak{u}^{\prime \prime}, \mathfrak{L}_{\mu+1}\right) \beta-\gamma_{p+1} \delta^{p}\left(\mathfrak{U}^{\prime \prime}, \mathfrak{\complement}_{\mu+1}\right) \beta\right) .
$$

Then $\delta^{p-1}\left(\mathfrak{U}^{\prime}, \varrho_{\mu}\right) \theta=\tau^{p}\left(\mathfrak{U}^{\prime}, \mathfrak{M}, \varrho_{\mu}\right)$. (D) ${ }_{\mu}$ is proved.

Fix $E^{\prime} \subset \subset E$ and $0 \leq \mu<n$. To finish the proof, we have to show that $\alpha_{E^{\prime}, E}^{(\mu)}$ exists. Take $E^{\prime} \subset \subset E^{\prime \prime} \subset \subset E^{\prime \prime \prime} \subset \subset E$. We can find $U_{i} \in \mathcal{G}, 1 \leq i \leq k$, such that $E^{\prime \prime \prime}=\bigcup_{i=1}^{k} U_{i}$ and such that $\alpha_{U_{i}, E}^{(\mu)}$ exists. Let $\mathfrak{U}=\left\{U_{i}\right\}_{i=1}^{k}$. Let $\alpha_{i}=$ $\phi_{U_{i}}^{(\mu+1)} \alpha_{U_{i}, E}^{(\mu)}$. Define $\sigma: \Gamma\left(E, \mathfrak{\varrho}_{\mu}\right) \rightarrow Z^{1}\left(\mathfrak{U}, \mathcal{S}_{\mu}\right)$ by

$$
\sigma(f)_{i j}=\left(\alpha_{j}(f)-\alpha_{i}(f)\right) \mid U_{i} \cap U_{j}
$$

for $1 \leq i, j \leq k$. By (D) ${ }_{\mu}$ there exists a finite covering $\mathfrak{U}^{\prime}=\left\{U_{i}^{\prime}\right\}_{i=1}^{l}$ of $E^{\prime \prime}$ by members of $\mathcal{E}$ which refines $\mathfrak{U}$ by means of an index map $r:\{1, \ldots, l\} \rightarrow\{1, \ldots, k\}$ and there exists a continuous linear map $\zeta: Z^{1}\left(\mathfrak{U}, \varrho_{\mu}\right) \rightarrow C^{0}\left(\mathfrak{U}^{\prime}, \varrho_{\mu}\right)$ such that $\delta^{0}\left(\mathfrak{u}^{\prime}, \mathfrak{S}_{\mu}\right) \zeta=\tau^{1}\left(\mathfrak{u}^{\prime}, \mathfrak{u}, \mathfrak{S}_{\mu}\right)$. Define $\lambda: \Gamma\left(E, \mathfrak{\varrho}_{\mu}\right) \rightarrow \Gamma\left(E^{n}, \mathfrak{S}_{\mu}\right)$ by

$$
\lambda(f) \mid U_{i}^{\prime}=\left(\alpha_{\tau(i)}(f) \mid U_{i}^{\prime}\right)-\zeta \sigma(f)_{i}
$$

for $1 \leq i \leq l$. By $(C)_{\mu}, \beta_{E^{\prime}, E^{n}}^{(\mu)}$ exists. The map $\alpha_{E^{\prime}, E}^{(\mu)}=\beta_{E^{\prime}, E^{(\mu)}}^{\lambda}$ satisfies the requirement. Q.E.D.

(3.5) Corollary. If, in addition to all the assumptions of Proposition (3.4), $X$ is compact and $X \in \mathcal{E}$, then the sequence of Fréchet spaces

$$
0 \rightarrow \Gamma\left(x,{\stackrel{\odot}{\varkappa_{n}}}_{n} \rightarrow \cdots \rightarrow \Gamma\left(x, \stackrel{\rho}{\llcorner}_{1}\right) \stackrel{\psi}{\rightarrow} \Gamma\left(x, \mathfrak{\varrho}_{0}\right)\right.
$$

induced by the sequence in Proposition (3.4) is direct-exact. Moreover, $\operatorname{Im} \psi$ is topologically isomorpbic to $\Gamma\left(X, \operatorname{Im} \phi^{(1)}\right)$.

4.

(4.1) For $a \in \mathrm{N}_{*}^{n}$ let $a_{1}, \ldots, a_{n}$ denote the components of $a$, let $D^{a}$ de- 


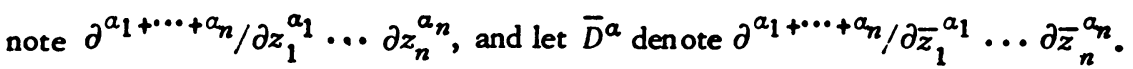

For a bounded polydomain $G=G_{1} \times \cdots \times G_{n}$ in $\mathbf{C}^{n}$, denote by $\Lambda(G)$ the set of all infinitely differentiable (complex-valued) functions $f$ on $G$ such that, if $\alpha, \beta \in \mathbf{N}_{*}^{n}$, then $D^{\alpha} \bar{D}^{\beta} f$ is uniformly continuous on any polydomain of the form $G_{1}^{\prime} \times \cdots \times G_{n}^{\prime}$, where $G_{i}^{\prime}=G_{i}$ for $i$ satisfying $\alpha_{i}=0$ and $G_{j}^{\prime} \subset \subset G_{j}$ for $j$ satisfying $a_{j}>0 . \Lambda(G)$ is a Fréchet space.

Denote by $\Lambda_{q}^{p}(G)$ the set of all $(0, p)$-forms on $G$ of the form

$$
\sum_{1 \leq i_{1}<\cdots<i_{p} \leq q} \xi_{i_{1} \cdots i_{p}} d \bar{z}_{i_{1}} \wedge \cdots \wedge d \bar{z}_{i_{p}}
$$

where $\xi_{i_{1} \ldots i_{p}}$ belongs to $\Lambda(G)$ and is holomorphic in $z_{q+1}, \ldots, z_{n}$ for fixed $z_{1}, \ldots, z_{q^{\cdot}} \Lambda_{q}^{p}(G)$ is a Fréchet space.

(4.2) Lemma. If $G$ is a bounded domain in $\mathrm{C}$, then there exists a continuous linear map $I: \Lambda(G) \rightarrow \Lambda(G)$ such that $(\partial / \partial \bar{z})$ I is the identity map of $\Lambda(G)$, where $z$ is the coordinate of $\mathrm{C}$.

Proof. For $f \in \Lambda(G)$ define $I(f)$ by

$$
\mu(f)(z)=\frac{1}{2 \pi i} \iint_{G} \frac{f(\zeta) d \zeta \wedge d \bar{\zeta}}{\zeta-z} \quad(z \in G) .
$$

We are going to prove that $l$ satisfies the requirements.

Let $R$ be a real number $>1$ such that $G$ is contained in the open disc of radius $(R-1) / 2$ centered at 0 . Let $B$ be the open disc of radius $R$ centered at 0 . For $c \geq 0$ let $b(c)$ be the supremum of $\iint_{E}|d \zeta \wedge d \bar{\zeta}| /|\zeta|$ as $E$ runs through all measurable sets of measure $\leq c$ in $B$. Since $\iint_{B}|d \zeta \wedge d \bar{\zeta}| /|\zeta|<\infty$, the limit of $b(c)$ is 0 as $c$ approaches 0 .

First we show that $I(f)$ is infinitely differentiable. Let $\left\{G_{m}\right\}$ be an increasing sequence of relatively compact open subsets of $G$ such that $U_{m} G_{m}=G$ and $\partial G_{m}$ is the disjoint union of a finite number of simple rectifiable closed curves in G. Define $I_{m}(f)$ by

$$
I_{m}(f)(z)=\frac{1}{2 \pi i} \iint_{G_{m}} \frac{f(\zeta) d \zeta \wedge d \bar{\zeta}}{\zeta-z} \quad(z \in G)
$$

Let $\mu_{m}$ be the measure of $G-G_{m}$. Then $\left\|I(f)-I_{m}(f)\right\|_{G} \leq b\left(\mu_{m}\right)\|f\|_{G}$, where $\|\cdot\|_{G}$ is the sup norm on $G$. A trivial modification of $[4$, p. 25, I.D. 2] shows that $I_{m}(f)$ is infinitely differentiable on $G_{m}$ and $(\partial / \partial \bar{z})_{m}(f)=f$ on $G_{m}$. For $k \geq$ $m,(\partial / \partial \bar{z})\left(I_{k}(f)-I_{m}(f)\right)=0$ on $G_{m}$ and hence $I_{k}(f)-I_{m}(f)$ is holomorphic on $G_{m}$. 
Since $\mu_{k} \rightarrow 0$ as $k \rightarrow \infty, I_{k}(f)$ approaches $I(f)$ uniformly on $G$ as $k \rightarrow \infty$. Hence for a fixed $m, I_{k}(f)-I_{m}(f)$ approaches $I(f)-I_{m}(f)$ uniformly on $G$ as $k \rightarrow \infty$. It follows that $I(f)-I_{m}(f)$ is holomorphic on $G_{m}$ for every $m$. Consequently $I(f)$ is infinitely differentiable and $(\partial / \partial \bar{z})(f)=(f)$ on $G$.

Next we have to show that $\left(\partial^{k} / \partial \bar{z}^{k}\right)(\lambda)$ is uniformly continuous on $G$ for every fixed $k \geq 0$. When $k \geq 1$, this is clear, because $(\partial / \partial \bar{z})(f)=f$ on $G$. So we need only show that $I(f)$ is uniformly continuous on $G$. Suppose $\epsilon>0$. By the uniform continuity of $f$ on $G$, there exists $0<\delta<1$ such that, if $a \in \mathbf{C}$ and $|a|<\delta$, then $|f(\zeta+a)-f(\zeta)|<\epsilon$ for $\zeta, \zeta+a \epsilon G$. We can also assume that $\delta$ is so chosen that, for $|a|<\delta$, the measure of $(G(a)-G) \cup(G-G(a))$ is $\leq$ some nonnegative number $c$ satisfying $b(c)<\epsilon$, where $G(a)=\{z \in \mathbf{C} \mid z=w-a$ for some $w \in G\}$. Then for $|a|<\delta$ and $z, z+a \in G$, we have

$$
|I(f)(z+a)-I(f)(z)|<\frac{\epsilon}{2 \pi}\left(\|f\|_{G}+\iint_{B} \frac{|d \zeta \wedge d \bar{\zeta}|}{|\zeta|}\right)
$$

because

$$
\begin{aligned}
I(f)(z+a)-I(f)(z)=\frac{1}{2 \pi i} & \left(\int_{G(a) \cap G} \frac{(f(\zeta+a)-f(\zeta)) d \zeta \wedge d \bar{\zeta}}{\zeta-z}\right. \\
& \left.\quad+\int_{G(a)-G} \frac{f(\zeta+a) d \zeta \wedge d \bar{\zeta}}{\zeta-z}+\iint_{G-G(a)} \frac{f(\zeta) d \zeta \wedge d \bar{\zeta}}{\zeta-z}\right) .
\end{aligned}
$$

Therefore $I(f)$ is uniformly continuous on $G$.

Finally we have to show that $I$ is continuous. Suppose a sequence $f_{k} \rightarrow 0$ in $\Lambda(G)$ as $k \rightarrow \propto$. We have to prove the following two statements.

(i) Every partial derivative of $I\left(f_{k}\right)$ converges to 0 uniformly on every compact subset of $G$ as $k \rightarrow \infty$

(ii) For every fixed $m \geq 0, \partial^{m} I\left(f_{k}\right) / \partial \bar{z}^{m}$ converges to 0 uniformly on $G$ as $k \rightarrow \infty$.

Statement (ii) follows from $\partial I\left(f_{k}\right) / \partial \bar{z}=f_{k}$ and

$$
\left\|I\left(f_{k}\right)\right\|_{G} \leq\left\|f_{k}\right\|_{G} \iint_{B} \frac{|d \zeta \wedge d \bar{\zeta}|}{|\zeta|}
$$

To prove statement (i), let $F$ be the Fréchet space of all infinitely differentiable (complex-valued) functions on $G$. Since $\partial_{/}^{\prime} \partial \bar{z}$ maps $F$ continuously onto $F$, there exists a sequence $\left\{g_{k}\right\}$ in $F$ such that $\partial g_{k} / \partial \bar{z}=f_{k}$ on $G$ and $g_{k} \rightarrow 0$ in $F$ as $k \rightarrow \infty$. By a trivial modification of $[4$, P. 24, I.D. 1$]$, 


$$
g_{k}(z)=\frac{1}{2 \pi i} \int_{\partial G_{m}} \frac{g_{k}(\zeta) d \zeta}{\zeta-z}+I_{m}\left(f_{k}\right)(z)
$$

for $z \in G_{m}$. Hence for a fixed $m$, every partial derivative of $I_{m}\left(f_{k}\right)$ converges to 0 uniformly on every compact subset of $G_{m}$ as $k \rightarrow \infty$. Since $\left\|I\left(f_{k}\right)-I_{m}\left(f_{k}\right)\right\|_{G}$ $\leq b\left(\mu_{m}\right) \max _{p}\left\|f_{p}\right\|_{G}$, the sequence $I\left(f_{k}\right)-I_{m}\left(f_{k}\right)$ approaches 0 uniformly on $G$ and uniformly in $k$ as $m \rightarrow \infty$. Since $I\left(f_{k}\right)-I_{m}\left(f_{k}\right)$ is holomorphic on $G_{m}$, every partial derivative of $I\left(f_{k}\right)-I_{m}\left(f_{k}\right)$ approaches 0 uniformly on every compact subset of $G_{m}$ and uniformly in $k$ as $m \rightarrow \infty$. Statement (i) follows. Q.E.D.

(4.3) Lemma. If $G$ is a polydomain in $\mathrm{C}^{n}$ and $1 \leq q \leq n$, then there exists a continuous linear map $J_{q}: \Lambda(G) \rightarrow \Lambda(G)$ such that $\left(\partial / \partial \bar{z}_{q}\right) J_{q}$ is the ident ity map of $\Lambda(G)$ and $J_{q}$ commutes with $\partial / \partial \bar{z}_{i}$ for $1 \leq i \leq n$ and $i \neq q$.

Proof. We can assume w.1.o.g. that $q=1$. Let $G=G_{1} \times \cdots \times G_{n}$, where $G_{i} \subset \mathrm{C}(1 \leq i \leq n)$. Let $G^{\prime}=G_{2} \times \cdots \times G_{n}$. For any function $f$ on $G$ and for any $z^{\prime} \in G^{\prime}$, denote by $f_{z^{\prime}}$ the function on $G_{1}$ defined by $f_{z^{\prime}}\left(z_{1}\right)=f\left(z_{1}, z^{\prime}\right)$ for $z_{1} \in G_{1}$.

By Lemma (4.2) there exists a continuous linear map $I: \Lambda\left(G_{1}\right) \rightarrow \Lambda\left(G_{1}\right)$ such that $\left(\partial / \partial \bar{z}_{1}\right) l$ is the identity map of $\Lambda\left(G_{1}\right)$. For $f \in \Lambda(G)$ define $J_{1}(f)$ by $J_{1}(f)_{z^{\prime}}=I\left(f_{z^{\prime}}\right)$ for $z^{\prime} \in G^{\prime}$. It is straightforward to verify that $J_{1}$ satisfies the requirements. Q.E.D.

(4.4) Proposition. If $G$ is a bounded polydomain in $\mathrm{C}^{n}$ and $0<p \leq q \leq n$, then there exists a continuous linear map $I_{q}^{p}$ from the kernel $Z_{q}^{p}$ of $\bar{\partial}: \Lambda_{q}^{p}(G)$ $\rightarrow \Lambda_{q}^{p+1}(G)$ to $\Lambda_{q}^{p-1}$ such that $\bar{\partial} l_{q}^{p}$ is the identity map of $Z_{q}^{p}$.

Proof. We use induction on $q$ for $0 \leq q \leq n$. The case $q=0$ is vacuous. For $q>0$, there exists $J_{q}$ satisfying the conditions of Lemma (4.3). Define a map $T: Z_{q}^{p} \rightarrow \Lambda_{q}^{p-1}(G)$ as follows. For

$$
\begin{aligned}
\xi & =\sum_{1 \leq i_{1}<\cdots<i_{p} \leq q} \xi_{i_{1} \cdots i_{p}} d \bar{z}_{i_{1}} \wedge \cdots \wedge d \bar{z}_{i_{p}} \in Z_{q}^{p}, \\
T(\xi) & =\sum_{1 \leq i_{1}<\cdots<i_{p-1} \leq q}(-1)^{p-1} J_{q}\left(\xi_{i_{1} \cdots i_{p-1} q}\right) d \bar{z}_{i_{1}} \wedge \cdots \wedge d \bar{z}_{i_{p-1}} .
\end{aligned}
$$

The map $I_{q}^{p}: Z_{q}^{p} \rightarrow \Lambda_{q}^{p-1}(G)$ defined by

$$
I_{q}^{p}(\xi)=\left\{\begin{array}{l}
T(\xi) \quad \text { if } p=q \\
I_{q-1}^{p}(\xi-\bar{\partial} T(\xi))+T(\xi) \quad \text { if } p<q
\end{array}\right.
$$

satisfies the requirement. Q.E.D. 
(4.5) Suppose $G$ is an open subset of $C^{n}$. Denote by $C_{G^{-}}$the sheaf on $G^{-}$ defined by the following presheaf:

(i) For every open subset $U$ of $G^{-}, f \in \Theta_{G^{-}}(U)$ if and only if $f$ is a continuous function on $U$ and $f$ is holomorphic on $G \stackrel{G^{-}}{\cap}$.

(ii) For open subsets $U^{\prime} \subset U$ of $G^{-}, \theta_{G^{-}}(U) \rightarrow \theta_{G^{-}}\left(U^{\prime}\right)$ is the restriction map.

The sheaf $\Theta_{G^{-}}$is a Fréchet sheaf.

Denote by $\mathcal{E}\left(G^{-}\right)$the superbasis for the topology of $G^{-}$defined as follows. $E \in \mathcal{E}\left(G^{-}\right)$if and only if $E=G^{-} \cap \widetilde{E}$ for some polydomain $\widetilde{E}$ in $\mathbf{C}^{n}$.

(4.6) Proposition. If $G$ is a bounded polydomain in $\mathrm{C}^{n}$, then the Fréchet sheaf $\mathcal{O}_{G^{-}}$on $G^{-}$is constructively fine relative to $\mathcal{E}\left(G^{-}\right)$.

Proof. Suppose $\mathfrak{U}=\left\{U_{i}\right\}_{i=1}^{k}$ and $\mathbb{B}=\left\{V_{i}\right\}_{i=1}^{k}$ are coverings of $G^{-}$by members of $\mathcal{G}\left(G^{-}\right)$such that $V_{i} \subset C U_{i}$ for $1 \leq i \leq k$. Let $W_{i}=G \cap V_{i}(1 \leq i \leq k)$ and $\mathbb{R}=\left\{W_{i}\right\}_{i=1}^{k}$. Let $C_{0}^{q}\left(\mathscr{B},{ }_{n} \mathcal{C}\right)$ be the set of all $\xi \in C^{q}\left(\mathbb{B},{ }_{n} \mathcal{E}\right)$ such that $\xi_{i_{0} \cdots i_{q}} \in B\left(W_{i_{0}} \cap \ldots \cap W_{i_{q}}\right)^{n}$. Let $\mathbb{Q}^{(p)}$ be the sheaf of germs of infinitely dif-

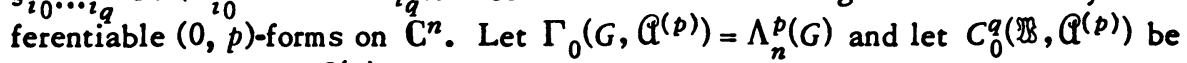
the set of $\eta \in C^{q}\left(\mathbb{B}, \mathbb{Q}^{(p)}\right)$ such that $\eta_{i_{0} \cdots i_{q}} \in \Lambda_{n}^{p}\left(W_{i_{0}}^{n} \cap \ldots \cap W_{i_{q}}\right)$. Consider the following commutative diagram of Fréchet spaces:

$$
\begin{aligned}
& \begin{array}{ll}
0 & 0 \\
\downarrow & \downarrow
\end{array} \\
& \Gamma_{0}\left(G, \mathbb{Q}^{(0)}\right) \rightarrow \Gamma_{0}\left(G, \mathbb{Q}^{(1)}\right) \rightarrow \cdots \\
& \text { ? } \\
& 0 \rightarrow C_{0}^{0}\left(\mathbb{B S},{ }_{n}(\mathrm{)}) \hookrightarrow C_{0}^{0}\left(\mathbb{B}, \mathbb{Q}^{(0)}\right) \rightarrow C_{0}^{0}\left(\mathbb{B}, \mathbb{Q}^{(1)}\right) \rightarrow \ldots\right. \\
& \downarrow \downarrow \downarrow \downarrow \\
& 0 \rightarrow C_{0}^{1}\left(\mathbb{B},{ }_{n} \theta\right) \subset C_{0}^{1}\left(\mathbb{B}, \mathbb{Q}^{(0)}\right) \rightarrow C_{0}^{1}\left(\mathbb{B}, \mathbb{Q}^{(1)}\right) \rightarrow \ldots \\
& \downarrow \quad \downarrow \quad \downarrow
\end{aligned}
$$

where (except the inclusion maps) all the horizontal maps are induced by $\partial$ and all the vertical maps are coboundary maps. By Proposition (4.4) all the rows are direct-exact. From the usual argument using a partition of unity, it follows that all the columns except the first are direct-exact. By diagram-chasing, we conclude that the first column is also direct-exact. Hence, for $r \geq 1$ there exists a continuous linear map $\psi: Z^{r}\left(\mathfrak{U}, \mathcal{C}_{G^{-}}\right) \rightarrow C^{r-1}\left(\mathfrak{B}, \mathcal{O}_{G^{-}}\right)$such that $\delta \psi$ is the restriction map $Z^{r}\left(\mathfrak{U}, \mathcal{C}_{G^{-}}\right) \rightarrow Z^{r}\left(\mathfrak{B}, \mathcal{C}_{G^{-}}\right)$, where $\delta: C^{r-1}\left(\mathfrak{B}, \Theta_{G^{-}}\right) \rightarrow Z^{r}\left(\mathfrak{B}, \mathcal{O}_{G^{-}}\right)$ is the coboundary map. Q.E.D. 
(4.7) Corollary. If $G$ is a bounded polydomain in $\mathrm{C}^{n}$, then $H^{p}\left(G^{-}, \Theta_{G^{-}}\right)=$ 0 for $p \geq 1$.

5.

(5.1) Lemma. Suppose $G_{1} \subset C G_{2}$ are Stein open subsets of $C^{n}$ and $\phi$ : ${ }_{n}{ }^{p} \rightarrow{ }_{n} \Theta^{q}$ is a sheaf-homomorphism on $G_{2}$. Then there exists a continuous linear map a: $\Gamma\left(G_{2},{ }_{n}{ }^{q}\right) \rightarrow \Gamma\left(G_{1},{ }_{n}{ }^{p}\right)$ sucb that $\phi_{1} a \phi_{2}=r \phi_{2}$, where $\phi_{i}$ : $\Gamma\left(G_{i},{ }_{n} \mathcal{O}^{p}\right) \rightarrow \Gamma\left(G_{i},{ }_{n} \mathcal{O}^{q}\right)$ is induced by $\phi(i=1,2)$ and $r: \Gamma\left(G_{2},{ }_{n}{ }^{q}\right) \rightarrow$ $\Gamma\left(G_{1},{ }_{n}^{q}\right)$ is the restriction map.

Proof. Let $G_{3}$ be a Stein open subset of $C^{n}$ satisfying $G_{1} \subset C G_{3} \subset C G_{2}$. Let $\Gamma_{*}\left(G_{i}{ }_{n} \Theta^{k}\right)$ be the set of all $k$-tuples of square-integrable holomorphic functions on $G_{i}(i=1,3 ; k=p, q)$. Let $\phi^{*}: \Gamma_{*}\left(G_{3},{ }_{n} \mathcal{E}^{q}\right) \rightarrow \Gamma_{*}\left(G_{3},{ }_{n} \mathcal{O}^{p}\right)$ be induced by $\phi$. Let $\left(\operatorname{Im} \phi^{*}\right)^{-}$be the topological closure of $\operatorname{Im} \phi^{*}$ in $\Gamma_{*}\left(G_{3},{ }_{n}{ }^{q}\right)$. By the closure-of-modules theorem [4, P. 85, II.D.3], (Im $\left.\phi^{*}\right)^{-}$is a subset of $\Gamma\left(G_{3}, \operatorname{Im} \phi\right)$. Consider the following commutative diagram

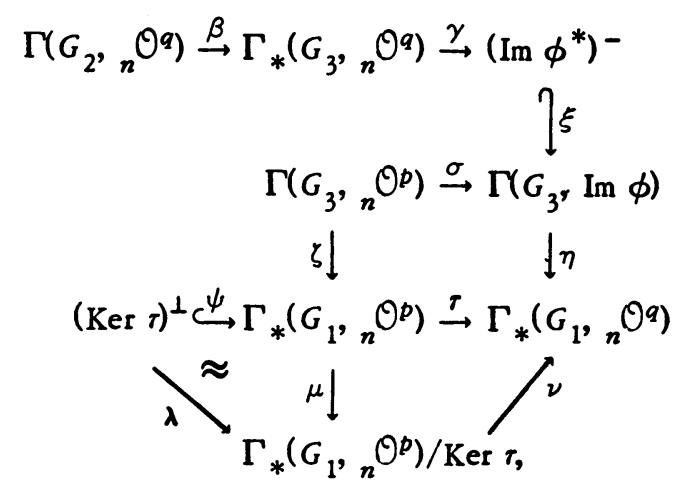

where $\gamma$ is the orthogonal projection; $\beta$ and $\zeta$ are restriction maps; $\eta$ is induced by restriction and by the inclusion map $\operatorname{Im} \phi \hookrightarrow{ }_{n}{ }^{q} ; \sigma$ and $\tau$ are induced by $\phi$; $(\operatorname{Ker} \tau)^{\perp}$ is the orthogonal complement of $\operatorname{Ker} \tau$ in $\Gamma_{*}\left(G_{1},{ }_{n}{ }^{p}\right) ; \mu$ is the quotient map; $\lambda$ is induced by $\mu$; and $\nu$ is induced by $r$. Since $\sigma$ is surjective, by the open mapping theorem for Fréchet spaces, $\nu^{-1} \eta$ is well defined and continuous. Let $\rho: \Gamma_{*}\left(G_{1},{ }_{n}{ }^{p}\right) \rightarrow \Gamma\left(G_{1},{ }_{n}{ }^{\rho}\right)$ be the inclusion map. Then $a=\rho \psi \lambda^{-1} \eta^{-1} \nu \xi \gamma \beta$ satisfies the requirement. Q.E.D.

(5.2) Lemma. Suppose $\Omega$ is a Stein open subset of $\mathbf{C}^{n}$ and

$$
0 \rightarrow \Theta^{\Theta^{p_{m}}} \rightarrow \ldots \rightarrow{ }_{n}^{\Theta^{p_{1}} \stackrel{\phi}{\rightarrow}}{ }_{n}^{\Theta^{p_{0}}}
$$

is an exact sequence on $\Omega$. Suppose $G$ is a polydomain in $\mathbf{C}^{n}$ and $G \subset \subset \Omega$. If $G$ 
is locally strongly (Im $\phi$ )-privileged, then the sequence

$$
0 \rightarrow \Theta_{G^{-}}^{p_{m}} \rightarrow \cdots \rightarrow \Theta_{G^{-}}^{p_{1}} \rightarrow \Theta_{G^{-}}^{p_{0}}
$$

is exact on $G^{-}$.

Proof. Let $\varrho=\operatorname{Im} \phi$. Take $x \in G^{-}$. Clearly (*) is exact at $x$ if $x \in G$. Assume that $x \in \partial G$. There exists a basis $\mathfrak{U}$ of open neighborhoods of $x$ in $\mathbf{C}^{n}$ such that $G \cap U$ is strongly $\mathcal{G}$-privileged for $U \in \mathfrak{U}$. The sequence

$$
0 \rightarrow B\left(G \cap U,{ }_{n}{ }^{p} m\right) \rightarrow \cdots \stackrel{a_{2}}{\longrightarrow} B\left(G \cap U,{ }_{n}{ }^{p} 1\right) \stackrel{a_{1}}{\longrightarrow} B\left(G \cap U,{ }_{n}{ }^{p} 0\right)
$$

is exact for $U \in \mathfrak{U}$ (Ker $\alpha_{1}=\operatorname{Im} \alpha_{2}$ follows from the injectivity of $B(G \cap U, \mathscr{G}) \rightarrow$ $\Gamma(G \cap U, \mathcal{G}))$. The lemma follows from the isomorphism ind $\lim _{U \in \mathfrak{U}} B\left(G \cap U,{ }_{n} \Theta^{p} i\right)$ $\approx\left(\Theta_{G^{-}}^{p_{i}}\right)_{x}$ for $0 \leq i \leq m$. Q.E.D.

(5.3) Proposition. Suppose $\Omega$ is a Stein open subset of $\mathbf{C}^{n}$ and $\mathcal{F}$ is a coberent analytic sheaf on $\Omega$ admitting a finite free resolution on $\Omega$. Suppose $G$ is a polydomain in $\mathrm{C}^{n}$ and $G \subset C \Omega$. If $G$ is locally strongly $\mathcal{F}$-privileged, then $G$ is strongly F-privileged.

Proof. Let $0 \rightarrow{ }_{n} \Theta^{p_{m}} \rightarrow \cdots \rightarrow{ }_{n} \mathcal{O}^{p_{1}} \rightarrow{ }_{n} \mathcal{O}^{p_{0}} \rightarrow \mathfrak{F} \rightarrow 0$ be the finite free resolution of $\mathcal{F}$ on $\Omega$ and let

$$
0 \rightarrow \Theta_{G^{-}}^{p_{m}} \stackrel{\phi^{(m)}}{\longrightarrow} \cdots \stackrel{\phi^{(2)}}{\longrightarrow} \Theta_{G^{-}}^{p_{1}} \stackrel{\phi^{(1)}}{\longrightarrow} \Theta_{G^{-}}^{p_{0}}
$$

be induced by it. By Lemma (5.2), (*) is exact.

Let $\mathbb{E}=\mathcal{E}\left(G^{-}\right)$. For $E \in \mathcal{E}$ and $1 \leq \mu \leq m$, denote by $\phi_{E}^{(\mu)}$ the map $\Gamma\left(E, \Theta_{G^{-}}^{p_{\mu}}\right)$ $\rightarrow \Gamma\left(E, \Theta_{G^{-}}^{p \mu-1}\right)$ induced by $\phi^{(\mu)}$. For $E^{\prime} \subset E$ in $E$ and $0 \leq \mu \leq m$, denote the restriction map $\Gamma\left(E, \Theta_{G^{-}}^{p_{\mu}}\right) \rightarrow \Gamma\left(E^{\prime}, \mathcal{O}_{G^{-}}^{p_{\mu}}\right)$ by $\rho_{E^{\prime}, E^{*}}^{(\mu)}$ We are going to prove that (*) is locally direct relative to $\mathcal{E}$.

Suppose $x \in G^{-}$and $E \in \mathcal{G}$ such that $x \in E$. We have to show that there exists $E^{\prime} \in \mathscr{G}$ with $x \in E^{\prime} \subset E$ and for $0 \leq \mu<m$ there exists a continuous linear $\operatorname{map}_{F^{\prime}, E} \alpha^{(\mu)} \Gamma\left(E, \Theta_{G^{-}}^{p \mu}\right) \rightarrow \Gamma\left(E^{\prime}, \mathcal{O}_{G^{-}}^{\left.p_{\mu+1}\right)}\right.$ such that $\phi_{E^{\prime}}^{(\mu+1)} \alpha_{E^{\prime}, E}^{(\mu)} \phi_{E}^{(\mu+1)}=$ $\rho_{E^{\prime}, E^{\prime}}^{(\mu)} \phi_{E}^{(\mu+1)}$. We distinguish between two cases.

(i) $x \in G$. We can assume that $E \subset G$. Choose $E^{\prime} \in \mathcal{G}$ such that $x \in E^{\prime} \subset C$ $E$. Then the existence of $\alpha_{E^{\prime}, E}^{(\mu)}$ follows from Lemma (5.1).

(ii) $x \in \partial G$. There exists an open neighborhood $U$ of $x$ in $\mathbf{C}^{n}$ such that ( $G \cap U)^{-} \subset E$ and $G \cap U$ is strongly $\mathcal{F}$-privileged. Choose $E^{\prime} \in \mathcal{E}$ such that $x \in E^{\prime} \subset U$. We claim that $\alpha_{E^{\prime}, E}^{(\mu)}$ exists. Fix $0 \leq \mu<m$. Let $W=G \cap U$. Let 
$\beta: \Gamma\left(W^{-}, \Theta_{W^{-}}^{p \mu+1}\right) \rightarrow \Gamma\left(W^{-}, \Theta_{W^{-}}^{p}\right)$ be induced by $\phi^{(\mu+1)}$. Since $G \cap U$ is strongly $\mathcal{F}$-privileged, there exists a continuous linear map $\gamma: \Gamma\left(w^{-}, \Theta_{w^{-}}^{p}\right) \rightarrow \Gamma\left(W^{-}, \Theta_{w^{-}}^{p}\right)$ such that $\beta y \beta=\beta$. Let

$$
\sigma: \Gamma\left(E, \Theta_{G^{-}}^{p}\right) \rightarrow \Gamma\left(W^{-}, \Theta_{W^{-}}^{p}\right) \text { and } \quad \tau: \Gamma\left(W^{-}, \Theta_{W^{-}}^{p} \mu+1\right) \rightarrow \Gamma\left(E^{\prime}, \Theta_{G^{-}}^{p}\right)
$$

be restriction maps. Then $\alpha_{E^{\prime}, E}^{(\mu)}=\tau \gamma \sigma$ satisfies the requirement.

By Corollary (3.5) and Proposition (4.6), the sequence of Banach spaces

$$
0 \rightarrow \Gamma\left(G^{-}, O_{G^{-}}^{p_{m}}\right) \rightarrow \cdots \rightarrow \Gamma\left(G^{-}, \Theta_{G^{-}}^{p_{1}}\right) \stackrel{\theta}{\rightarrow} \Gamma\left(G^{-}, \Theta_{G^{-}}^{p_{0}}\right)
$$

is direct-exact and $\operatorname{Im} \theta=\Gamma\left(G^{-}, \operatorname{Im} \phi^{(1)}\right)$. Since $\Gamma\left(G^{-}, O_{G^{-}}^{p_{i}}\right)$ is topologically isomorphic to $B\left(G,{ }_{n} \Theta^{p_{i}}\right)$ for $0 \leq i \leq m, G$ is $\mathcal{F}_{\text {-privileged. }}$

Suppose $f \in \Gamma\left(G^{-}, \mathcal{O}_{G^{-}}^{p}\right)$ and $f \mid G \in \Gamma\left(G, \operatorname{Im} \phi^{(1)}\right)$. To prove that $G$ is

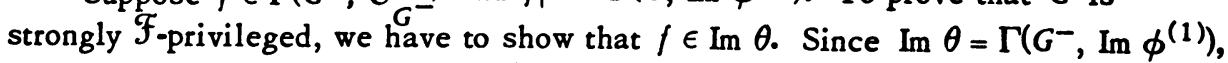
we need only show that $f_{x} \in\left(\operatorname{Im} \phi^{(1)}\right)_{x}$ for $x \in \partial G$. Take $x \in \partial G$. There exists an open neighborhood $U$ of $x$ in $\mathbf{C}^{n}$ such that $G \cap U$ is strongly $\mathfrak{F}_{\text {-privileged. }}$ Let $W=G \cap U$. Since $f\left|W \in \Gamma\left(W, \operatorname{Im} \phi^{(1)}\right), f\right| W^{-}$belongs to the image of the map $\Gamma\left(W^{-}, \mathcal{O}_{W^{-}}^{1}\right) \rightarrow \Gamma\left(W^{-}, \mathcal{O}_{W^{-}}^{p}\right)$ induced by $\phi^{(1)}$. Hence $f_{x} \in\left(\operatorname{Im} \phi^{(1)}\right)_{x^{\cdot}} \quad$ Q.E.D.

6.

(6.1) Lemma. Suppose $G$ is an open neighborbood of 0 in $\mathrm{C}^{n}$ and $V_{0}, \ldots$, $V_{n-1}$ are subvarieties in $G$ such that $\operatorname{dim} V_{k} \leq k(0 \leq k<n)$. Then there exists an open polydisc $P \neq \varnothing$ in $C^{n}$ centered at 0 such that $P \subset C G$ and $V_{k} \cap \partial_{k+1} P$ $=\varnothing$ for $0 \leq k<n$.

Proof. Use induction on $n$. The case $n=0$ is trivial. Identify $\mathrm{C}^{n-1}$ with $\mathrm{C}^{n} \cap\left\{z_{n}=0\right\}$. Let $V_{k-1}^{\prime}$ be the union of branches of $V_{i} \cap\left\{z_{n}=0\right\}$ of dimension $\leq k-1$. By induction hypothesis, there exists an open $(n-1)$-dimension polydisc $P^{\prime} \neq \varnothing$ centered at 0 such that $P^{\prime} \subset \subset G \cap\left\{z_{n}=0\right\}$ and $V_{k}^{\prime} \cap \partial_{k+1} P^{\prime}=\varnothing$ for $0^{\circ} \leq k<n-1$. For $\delta>0$ let $D_{\delta}$ be the open disc in $\mathbf{C}$ with center 0 and radius $\delta$. There exists $\delta>0$ such that $P^{\prime} \times D_{\delta} \subset C G$ and $W \cap\left(\left(\partial_{k+1} P^{\prime}\right) \times D_{\delta}^{-}\right)=\varnothing$ for any branch $W$ of $V_{k}$ not contained in $\left\{z_{n}=0\right\}$. Then $P=P^{\prime} \times D_{\delta}$ satisfies the requirement. Q.E.D.

(6.2) Proposition. Suppose $\Omega$ is a Stein open subset of $\mathbf{C}^{n}$ and $\mathcal{F}$ is a coberent analytic sheaf on $\Omega$ admitting a finite free resolution on $\Omega$. Suppose $G$ is a bounded polydomain in $\mathbf{C}^{n}$ and $G \subset \subset \Omega$. If $S_{k}(\mathcal{F}) \cap \partial_{k+1} G=\varnothing$ for $0 \leq k<n$, then $G$ is locally strongly $\mathcal{F}$-privileged and bence strongly $\mathcal{F}_{\text {-privileged. }}$

Proof. Use induction on $n$. The case $n=0$ is trivial. Take $x \in G$ and 
take an open neighborhood $U$ of $x$ in $\mathrm{C}^{n}$. Let $k$ be the largest integer such that $x \in \partial_{k} G$. We can assume w.1.o.g. that $x=\left(z_{1}^{0}, \ldots, z_{n}^{0}\right)$ with $z_{i}^{0} \in \partial G_{i}$ for $1 \leq$ $i \leq k$. Let $x^{\prime}=\left(z_{1}^{0}, \ldots, z_{k}^{0}\right)$ and let

$$
\mathcal{G}=\left(\mathcal{F} / \sum_{i=1}^{k}\left(z_{i}-z_{i}^{0}\right) \mathcal{F}\right) \mid\left\{x^{\prime}\right\} \times \mathrm{C}^{n-k} .
$$

By induction hypothesis and Lemma (6.1), there exists a nonempty open $(n-1)$. dimensional polydisc $P$ centered at $\left(z_{k+1}^{0}, \cdots, z_{n}^{0}\right)$ such that $P \subset G_{k+1} \times \cdots$

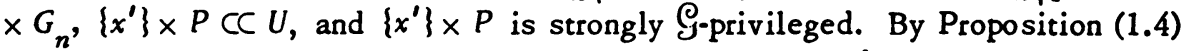
and (2.4), there exists an open neighborhood $D$ of $x^{\prime}$ in $C^{k}$ such that $D \times P \subset U$ and $\left(\left(G_{1} \times \cdots \times G_{k}\right) \cap D\right) \times P$ is strongly $\mathcal{F}_{\text {-privileged. } \quad \text { Q.E.D. }}$

(6.3) Lemma. Suppose $\Omega$ is an open subset of $\mathbf{C}^{n}$ and $V$ is a subvariety of pure dimension $k$ in $\Omega$. Suppose $G$ is a polydomain in $\mathrm{C}^{n}$ such that $G \subset C \Omega$. If $V \cap \partial_{k+1} G=\varnothing ;$ then $V \cap G$ bas only a finite number of branches.

Proof. The cases where $k \leq 0$ or $k \geq n$ are trivial. We can assume that $0<$ $k<n$. There exists $G_{i}^{\prime} \subset C G_{i}(1 \leq i \leq n)$ such that, for $1 \leq i \leq n, G_{i}-\left(G_{i}^{\prime}\right)-$ has only a finite number of components and, for any distinct element $s i_{1}, \cdots, i_{k+1}$ of $\{1, \cdots, n\}$, the set

$$
\left\{\left(z_{1}, \cdots, z_{n}\right) \in G^{-} \cap V \mid z_{i} \in G_{i}^{-}-G_{i}^{\prime} \text { for } i=i_{1}, \cdots, i_{k+1}\right\}
$$

is empty. Let

$$
D_{i_{1} \cdots i_{k}}=\left\{\left(z_{1}, \cdots, z_{n}\right) \in G \mid z_{i} \in G_{i}-\left(G_{i}^{\prime}\right)^{-} \text {for } i=i_{1}, \cdots, i_{k}\right\}_{0}
$$

Then, for any distinct elements $i_{1}, \ldots, i_{k}$ of $\{1, \ldots, n\}$, the projection $\left(z_{1}\right.$, $\left.\ddot{\cdots}, z_{n}\right) \mapsto\left(z_{i_{1}}, \ldots, z_{i_{k}}\right)$ makes $D_{i_{1} \cdots i_{k}} \cap V$ an analytic cover over $\left(G_{i_{1}}-\right.$ $\left.\left(G_{i_{1}}^{\prime}\right)^{-}\right) \times \cdots \times\left(G_{i_{k}}-\left(G_{i_{k}}^{\prime}\right)-\right)$. Hence, it suffices to show that every branch of $V \cap G$ intersects some $D_{i_{1} \cdots i_{k}}$.

Suppose $W$ is a branch of $V \cap G$. Let $G^{\prime}=G_{1}^{\prime} \times \cdots \times G_{n}^{\prime}$. We can choose a polydomain $G^{\prime \prime}$ in $C^{n}$ such that $G^{\prime} \subset C G^{\prime \prime} \subset C G$ and $W \cap\left(G^{\prime \prime}\right)-\not \varnothing$. By Lemma (1.3), $W \cap \partial_{k} G^{N} \neq 0$. Hence $W$ intersects some $D_{i_{1} \cdots i_{k}} \quad$ Q.E.D.

(6.4) Proposition. Suppose $\Omega$ is a Stein open subset of $\mathbf{C}^{n}$ and $\mathcal{F}$ is a coberent analytic sheaf on $\Omega$ admitting a finite free resolution on $\Omega$. Suppose $G$ is a polydomain in $\mathrm{C}^{n}$ and $G \subset \subset \Omega$. If $S_{k}(\mathcal{F}) \cap \partial_{k+1} G=\varnothing$ for $0 \leq k<n$, then $G$

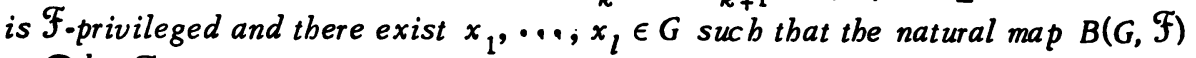
$\rightarrow \bigoplus_{i=1}^{l} \mathfrak{F}_{x_{i}}$ is injective.

Proof. By Lemma (6.3), we can choose $x_{1}, \cdots, x_{l} \in G$ such that, for $0 \leq$ 
$k<n$, every $k$-dimensional branch of $G \cap S_{k}(\mathcal{F})$ contains some $x_{i}$. The proposition follows from Proposition (6.2) and [7, p. 49, (2.3)]. Q.E.D.

7. For a holomorphic Banach bundle $E$, denote by $O(E)$ the sheaf of germs of holomorphic sections of $E$.

(7.1) Lemma. Suppose $\Omega$ is an open subset of $\mathrm{C}^{n}, E^{(i)}$ is a globally triv. ial bolomorphic Banacb bundle on $\Omega(0 \leq i \leq m)$, and

$$
0 \rightarrow E^{(m)} \stackrel{\phi^{(m)}}{\longrightarrow} \ldots \rightarrow E^{(1)} \stackrel{\phi^{(1)}}{\longrightarrow} E^{(0)}
$$

is a complex of bundle-bomomorphisms on $\Omega$. Let

$$
0 \rightarrow \Theta\left(E^{(m)}\right) \stackrel{\psi^{(m)}}{\longrightarrow} \cdots \rightarrow \Theta\left(E^{(1)}\right) \stackrel{\psi^{(1)}}{\longrightarrow} \Theta\left(E^{(0)}\right)
$$

be induced by (*). Suppose $G=G_{1} \times \cdots \times G_{n}$ is a polydomain in $\mathbf{C}^{n}$ such that $G \subset C \Omega$ and every point of $\partial G_{i}$ is a peak point of $B\left(G_{i}\right)$ for $1 \leq i \leq n$. Suppose the following three conditions are satisfied.

(i) For $1 \leq i \leq m$ and $z^{0} \in \partial_{n} G, \phi_{z^{0}}^{(i)}$ is direct.

(ii) For $1 \leq i \leq m$, the map $\left.B\left(G, E^{(i}\right)^{3}\right) \rightarrow B\left(G, E^{(i-1)}\right)$ induced by $\phi^{(i)}$ bas a closed image.

(iii) There exists an open neighborbood $D$ of $\partial_{n} G$ in $\Omega$ such that the complex (\#) is exact on D.

Then the complex $(*)$, when restricted to $\partial_{n} G$, is exact.

Proof. Use induction on $m$. The case $m=0$ is trivial. Assume $m>0$. Let $B^{*}\left(G^{-}, E^{(i)}\right)$ be the Banach space of all sections of $E^{(i)}$ over $G^{-}$which are continuous in $G^{-}$and holomorphic on $G(0 \leq i<m)$. Let $\tilde{\phi}^{(i)}: B^{*}\left(G^{-}, E^{(i-1)}\right) \rightarrow$ $B^{*}\left(G^{-}, E^{(i-1)}\right)$ be induced by $\phi^{(i)}(0<i \leq m)$. Since $B^{*}\left(G^{-}, E^{(i)}\right)$ is topologically isomorphic to $B\left(G, E^{(i)}\right)(0 \leq i \leq m), \operatorname{Im}{\widetilde{\phi^{(}}}^{(i)}$ is closed in $B^{*}\left(G^{-}, E^{(i-1)}\right)(0<i \leq m)$.

Fix $z^{0}=\left(z_{1}^{0}, \ldots, z_{n}^{0}\right) \in \partial_{n} G$. By induction hypothesis and (2.2), for some open neighborhood $U$ of $z^{0}$ in $D, \operatorname{Im} \phi^{(2)} \mid U$ is a holomorphic Banach bundle and there exists a holomorphic Banach bundle $F$ on $U$ such that

$$
E^{(1)} \mid U=F \oplus\left(\operatorname{Im} \phi^{(2)} \mid U\right) .
$$

Let $a: F \rightarrow E^{(0)} \mid U$ be the restriction of $\phi^{(1)}$. We need only show that (Ker $\left.a\right)_{z^{0}}$ $=0$. Suppose the contrary. Then there exists $e \in F_{z^{0}}$ such that $e \not \neq 0$ and $\alpha_{z_{0}}(e)=0$. Since $E^{(1)}$ is globally trivial, there exists $f \in B^{*}\left(G^{-}, E^{(1)}\right)$ such that $f\left(z^{0}\right)=e$. We have $\tilde{\phi}^{(1)}(f)\left(z^{0}\right)=\alpha_{z_{0}}(e)=0$. 
Since $z_{i}^{0}$ is a peak point of $B\left(G_{i}\right)$, there exists a function $\lambda_{i}$ on $G_{i}^{-}$which is continuous on $G_{i}^{-}$and is holomorphic on $G_{i}$ such that $\lambda_{i}\left(z_{i}^{0}\right)=1$ and $\left|\lambda_{i}\left(z_{i}\right)\right|<1$ for $z_{i} \in G_{i}^{-}-\left\{z_{i}^{0}\right\} \quad(1 \leq i \leq n)$. Let $\lambda=\lambda_{1} \ldots \lambda_{n}$. Let $H$ be the fiber of the trivial bundle $E^{(0)}$ and let $\|\cdot\|_{H}$ be the norm of $H$. The sequence $\tilde{\phi}^{(1)}\left(\lambda^{\mu} f\right)$ approaches 0 in $B^{*}\left(G^{-}, E^{(0)}\right)$ as $\mu \rightarrow \infty$, because, for any given $\epsilon>0$, there exists an open neighborhood $W$ of $z^{0}$ in $C^{n}$ such that $\left\|\tilde{\phi}^{(1)}(f)(z)\right\|_{H}<\epsilon$ for $z \in G^{-} \cap W$ and there exists $\mu_{0}$ such that

$$
\left(\sup _{z \in G^{-}-W}|\lambda(z)|\right)^{\mu}\left(\sup _{z \in G^{-}}\left\|\tilde{\phi}^{(1 Y}(f)(z)\right\|_{H}\right)<\epsilon
$$

for $\mu \geq \mu_{0}$. Since $\tilde{\phi}^{(1)}$ has a closed image, by applying the open mapping theorem to the map $B^{*}\left(G^{-}, E^{(1)}\right) \rightarrow \operatorname{Im} \tilde{\phi}^{(1)}$ induced by $\widetilde{\phi}^{(1)}$, we conclude that there exists $g_{\mu} \in B^{*}\left(G^{-}, E^{(1)}\right)(1 \leq \mu<\infty)$ such that $g_{\mu} \rightarrow 0$ in $B^{*}\left(G^{-}, E^{(1)}\right)$ as $\mu \rightarrow$ $\infty$ and $\mathscr{\phi}^{(1)}\left(g_{\mu}\right)=\check{\phi}^{(1)}\left(\lambda^{\mu} f\right)(1 \leq \mu<\infty)$.

Let $\beta: E^{(1)} \mid U \rightarrow F$ be the projection obtained from the direct sum decomposition ( $\dagger$ ). Let $\widetilde{\beta}: \mathcal{C}\left(E^{(1)}\right) \mid U \rightarrow \mathcal{O}(F)$ be induced by $\beta$. Since $\widetilde{\phi}^{(1)}\left(\lambda^{\mu} f-g_{\mu}\right)=0$, we have

$$
\left(\lambda^{\mu} f-g_{\mu}\right) \mid G \cap U \in \Gamma\left(G \cap U, \operatorname{Ker} \psi^{(1)}\right)
$$

for $1 \leq \mu<\infty$. Since the complex (\#) is exact in $D$,

$$
\left(\lambda^{\mu} f-g_{\mu}\right) \mid G \cap U \in \Gamma\left(G \cap U, \operatorname{Im} \psi^{(2)}\right)
$$

for $1 \leq \mu<\infty$. By $(\dagger), \mathcal{O}\left(\operatorname{Im} \phi^{(2)} \mid U\right)=\operatorname{Im} \psi^{(2)} \mid U$. Hence $\widetilde{\beta}\left(\left(\lambda^{\mu} f-g_{\mu}\right) \mid G \cap U\right)=0$ for $1 \leq \mu<\infty$. Since $\widetilde{\beta}\left(\left(\lambda^{\mu} f-g_{\mu}\right) \mid G^{-} \cap U\right)$ is a continuous section of $F$ over $G^{-} \cap \bar{U}$, we have $\widetilde{\beta}\left(\left(\lambda^{\mu} f-g_{\mu}\right) \mid G^{-} \cap U\right)=0$ for $1 \leq \mu<\infty$. Hence

$$
\beta_{z^{0}}\left(g_{\mu}\left(z^{0}\right)\right)=\lambda\left(z^{0}\right)^{\mu} \beta_{z}{ }\left(f\left(z^{0}\right)\right)=e \quad(1 \leq \mu<\infty) .
$$

Since $g_{\mu} \rightarrow 0$ in $B^{*}\left(G^{-}, E^{(1)}\right)$ as $\mu \rightarrow \infty$, we have $\beta_{z^{0}}\left(g_{\mu}\left(z^{0}\right)\right) \rightarrow 0$ as $\mu \rightarrow \infty$, contradicting that $e \neq 0$. Q.E.D.

(7.2) Lemma. Suppose $0 \rightarrow \mathfrak{G} \rightarrow \mathcal{F} \rightarrow R \rightarrow 0$ is an exact sequence of coberent analytic sheaves and sheaf-homomorphisms on a Stein complex space $(X, \mathcal{O})$. Suppose $\mathcal{G}$ and $\mathcal{F}$ bave finite free resolutions of length $\leq m$ on $X$. If $Y$ is a relatively compact Stein open subset of $X$, then there exists a finite free resolution of $R$ on $Y$ of lengtb $\leq m+1$.

Proof. First we make the following observation. If 


$$
\begin{aligned}
& 0 \rightarrow K \rightarrow \mathcal{O}^{\mu} l \rightarrow \ldots \rightarrow \mathcal{O}^{\mu} \rightarrow \mathfrak{T} \rightarrow 0, \\
& 0 \rightarrow K^{\prime} \rightarrow \mathcal{O}^{\mu} l \rightarrow \ldots \rightarrow \mathcal{O}^{\mu_{0}^{\prime}} \rightarrow \mathfrak{T} \rightarrow 0
\end{aligned}
$$

are two exact sequences of sheaf-homomorphisms on $X$, then there exist nonnegative integers $\mu$ and $\mu^{\prime}$ such that $K \oplus O^{\mu} \approx K^{\prime} \oplus O^{\mu^{\prime}}$ on $X$, because, by a trivial modification of the proof of [4, p. 202, VI. F. 3], we can show, by using Theorem B of Cartan-Oka, that the two sequences become isomorphic sequences after we apply to each of them a finite number of modifications [4, p. 201, Definition VI. F. 1].

Construct the following commutative diagram on $Y$ with exact rows and exact columns:

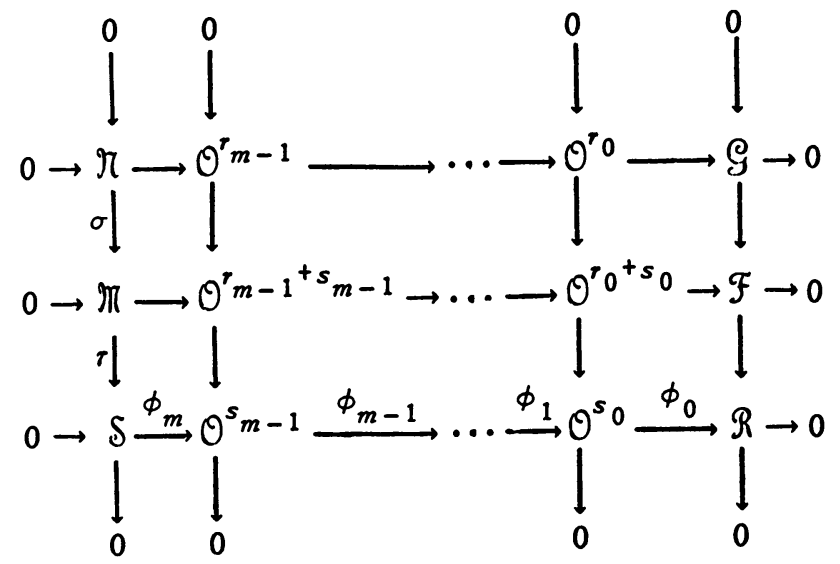

By the preceding observation, for some nonnegative integer $k, p$, and $q$, there exist sheaf-isomorphisms

$$
\alpha: r \oplus \Theta^{k} \stackrel{\approx}{\rightarrow} \Theta^{p} \text { and } \beta: \mathbb{R} \oplus \Theta^{k} \stackrel{\approx}{\rightarrow} \Theta^{q} \text { on } Y \text {. }
$$

Define $\gamma: \pi \oplus \mathcal{O}^{k} \rightarrow \pi \oplus \mathcal{O}^{k}$ by $\gamma(f \oplus g)=\sigma(f) \oplus g$ for $f \oplus g \in \pi_{x} \oplus \mathcal{O}_{x}^{k}$ and $x \in Y$.

We have the following finite free resolution of $R$ on $Y$ :

$$
\begin{aligned}
0 \rightarrow \Theta^{p} \stackrel{\tilde{\phi}_{m+1}}{\longrightarrow} \Theta^{a} \stackrel{\tilde{\phi}_{m}}{\longrightarrow} \theta^{s} m-1 \oplus \Theta^{k} \\
\stackrel{\tilde{\phi}_{m-1}}{\longrightarrow} \theta^{s-2} \stackrel{\phi_{m-2}}{\longrightarrow} \ldots \stackrel{\phi_{1}}{\longrightarrow} \Theta^{s_{0}} \stackrel{\phi_{0}}{\longrightarrow} R \rightarrow 0,
\end{aligned}
$$

where $\tilde{\phi}_{m+1}=\beta \gamma \alpha^{-1} ; \widetilde{\phi}_{m-1}(a \oplus \underset{\sim}{b})=\phi_{m-1}(a)$ for $a \oplus b \in \mathcal{O}_{x}^{s_{m-1}} \oplus \mathcal{O}_{x}^{k}$ and $x \in Y$; and $\tilde{\phi}_{m}$ is so defined that $\tilde{\phi}_{m} \beta(c \oplus d)=\phi_{m} \tau(c) \oplus d$ for $c \oplus d \in \mathbb{H}_{x} \oplus$ $\mathcal{O}_{x}^{k}$ for $x \in Y$. Q.E.D.

(7.3) Lemma. Suppose $S$ and $\Omega$ are respectively open subsets of $\mathrm{C}^{k}$ and 
$\mathrm{C}^{n}$, and $\pi: S \times \Omega \rightarrow S$ is the natural projection. Suppose

$$
0 \rightarrow{ }_{k+n} \mathcal{O}^{p} m \rightarrow \cdots \rightarrow_{k+n} \mathcal{O}^{p_{1}} \rightarrow_{k+n} \Theta^{p_{0}} \stackrel{\phi_{0}}{\longrightarrow} \mathcal{F} \rightarrow 0
$$

is an exact sequence of sheaf-homomorphisms on $S \times \Omega$. Suppose $s \in S$ and $K$ is a compact subset of $\Omega$ admitting a basis of Stein neigbborboods in $\Omega$. If $\operatorname{Ker} \phi_{0}$ is $\pi-f l a t$ on $\{s\} \times K$ and $\mathcal{F}$ is $\pi-f l a t$ on $\{s\} \times \partial K$, then, for some open neighborbood $\Omega^{\prime}$ of $K$ in $\Omega, \mathcal{F}(s)$ admits a finite free resolution on $\Omega^{\prime}$.

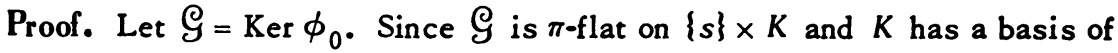
Stein neighborhoods in $\Omega$, there exists a Stein open neighborhood $\Omega^{n}$ of $K$ in $\Omega$ such that

$$
0 \rightarrow{ }_{k+n} \Theta^{p} m(s) \rightarrow \cdots \rightarrow k_{k+n} \Theta^{p} 1(s) \rightarrow S(s) \rightarrow 0
$$

is exact on $\Omega^{N}$. Hence $\mathcal{G}(s)$ admits a finite free resolution on $\Omega^{\prime \prime}$. Consider the sequence

$$
\mathcal{G}(s) \stackrel{a}{\rightarrow}{ }_{k+n} \Theta^{p} 0(s) \rightarrow \mathcal{F}(s) \rightarrow 0 .
$$

Since $\mathcal{F}$ is $\pi$-flat on $\{s\} \times \partial K$, the support of $\operatorname{Ker} a$ is disjoint from $\partial K$. For some relatively compact Stein open neighborhood $\Omega^{*}$ of $K$ in $\Omega^{\prime \prime}$, the support of Ker $\alpha \mid \Omega^{*}$ is a finite set. Ker $\alpha \mid \Omega^{*}$ can be trivially extended to a coherent analytic sheaf on $\mathbf{C}^{n}$. Hence $\operatorname{Ker} \alpha$ admits a finite free resolution on $\Omega^{*}$. By applying Lemma (7.2) to the two sequences on $\Omega^{*}$ :

$$
\begin{aligned}
& 0 \rightarrow \operatorname{Ker} a \rightarrow \mathcal{G}(s) \rightarrow \operatorname{Im} a \rightarrow 0, \\
& 0 \rightarrow \operatorname{Im} a \rightarrow{ }_{k+n} \Theta^{p} 0(s) \rightarrow \mathcal{F}(s) \rightarrow 0,
\end{aligned}
$$

we conclude that $\mathcal{F}(s)$ admits a finite free resolution on any relatively compact open neighborhood $\Omega^{\prime}$ of $K$ in $\Omega^{*}$. Q.E.D.

(7.4) Lemma. Suppose $\Omega$ is a Stein open subset of $\mathbf{C}^{n}$ and $\mathfrak{F}$ is a coberent analytic sheaf on $\Omega$ admitting a finite free resolution on $\Omega$. Suppose $G$ is a polydomain in $\mathrm{C}^{n}$ : such that $G \subset C \Omega$ and $G$ is $\mathcal{F}$-privileged. If ${ }_{n} \Theta^{q 1} \underset{\psi_{n}}{\rightarrow} \Theta^{q 0} \rightarrow \mathcal{F}$ is an exact sequence on $\Omega$, then the map $\widetilde{\psi}: B\left(G,{ }_{n} \mathcal{O}^{q 1}\right) \rightarrow{ }_{n}^{n}\left(G,{ }_{n}^{\Theta^{q} 0}\right)$ induced by $\psi$ is direct.

Proof. Let

$$
0 \rightarrow{ }_{n} \Theta^{p} m \rightarrow \cdots \rightarrow{ }_{n} \Theta^{p_{1}} \stackrel{\phi}{\rightarrow}{ }_{n} \Theta^{p_{0}} \rightarrow \mathcal{F} \rightarrow 0
$$


be the finite free resolution of $\mathcal{F}$ on $\Omega$. By a trivial modification of the proof of [4, p. 202, VI. F. 3], we can construct the following commutative diagram on $\Omega$ :

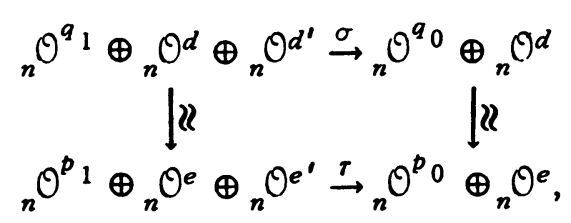

where $o(a \oplus b \oplus c)=\psi(a) \oplus b$ for $a \oplus b \oplus c \in{ }_{n} \mathcal{O}_{n}^{q 1} \oplus{ }_{n} \mathcal{O}_{x}^{d} \oplus_{n} \mathcal{O}_{x}^{d^{\prime}}$ and $x \in \Omega$; and $r(f \oplus g \oplus b)=\phi(f) \oplus g$ for $f \oplus g \oplus b \epsilon_{n} \mathcal{O}_{x}^{p_{1}} \oplus{ }_{n} \mathcal{O}_{x}^{e} \oplus{ }_{n} \mathcal{O}_{x}^{e^{\prime}}$ and $x \in \Omega$.

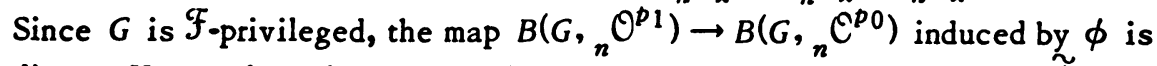
direct. Hence, from the commutative diagram above, we conclude that $\widetilde{\psi}$ is direct. Q.E.D.

(7.5) Lemma. Suppose $S$ and $\Omega$ are respectively Stein open subsets of $\mathbf{C}^{k}$ and $\mathrm{C}^{n}$ and $\mathcal{F}$ is a coberent analytic sheaf on $S \times \Omega$ admitting a finite free resolution

$$
0 \rightarrow{ }_{k+n} \Theta^{p_{m}} \rightarrow \cdots \rightarrow{ }_{k+n} \Theta^{p_{1}} \rightarrow{ }_{k+n} \Theta^{p_{0}} \rightarrow \mathcal{F} \rightarrow 0
$$

on $S \times \Omega$. Suppose $s \in S$ and $G$ is a relatively compact open subset of $\Omega$. If the sequence

$(*) \quad 0 \rightarrow B\left(G,{ }_{k+n} \Theta^{p} m(s)\right) \rightarrow \cdots \rightarrow B\left(G,{ }_{k+n} \Theta^{p}(s)\right) \rightarrow B\left(G,{ }_{k+n} \Theta^{p} 0(s)\right)$

is exact, then $\mathcal{F}$ is $\pi$-flat on $\{s\} \times G$, where $\pi: S \times \Omega \rightarrow S$ is the natural projection.

Proof. We need only show that the sequence

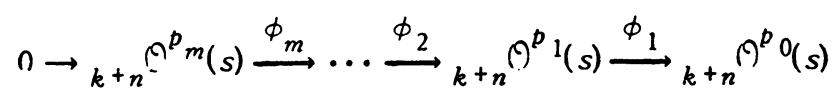

is exact on $G$. This follows from the exactness of $(*)$, because, for $1 \leq i \leq m$, $\Gamma\left(\Omega, \operatorname{Ker} \phi_{i}\right)$ generates $\operatorname{Ker} \phi_{i}$ on $\Omega$. Q.E.D.

(7.6) Proposition. Suppose $\Omega$ is a Stein open subset of $\mathrm{C}^{n}$ and $\mathcal{F}$ is a coberent analytic sheaf on $\Omega$ admitting a finite free resolution on $\Omega$. Suppose $G=G_{1} \times \cdots \times G_{n}$ is a polydomain in $\mathbf{C}^{n}$ such that $G \subset C \Omega$ and every point of $\partial G_{i}$ is a peak point of $B\left(G_{i}\right)$ for $1 \leq i \leq n$. If $G$ is weakly $\mathcal{F}_{\text {-privileged, then }}$ $S_{k}(\mathcal{F}) \cap \partial_{k+1} G=\varnothing$ for $0 \leq k<n$.

Proof. We can assume w.l.o.g. that $\Omega$ is a polydomain $\Omega_{1} \times \cdots \times \Omega_{n}$. Let 


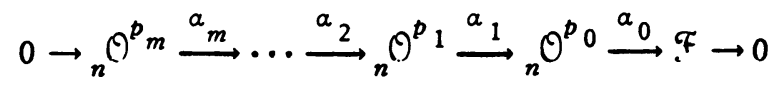

be the finite free resolution of $\mathcal{F}$ on $\Omega$. We are going to prove the proposition by induction on $m$. The case $m=0$ is trivial. Suppose $m>0$. Let $\mathcal{G}=\operatorname{Ker} a_{0}$.

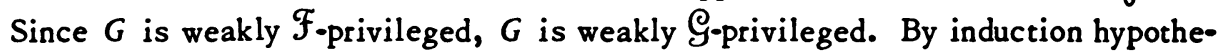
sis, $S_{k}(\biguplus) \cap \partial_{k+1} G=\varnothing$ for $0 \leq k<n$.

For distinct elements $i_{1}, \cdots, i_{k}$ of $\{1, \cdots, n\}$ let $\pi_{i_{1} \cdots i_{k}}: \mathrm{C}^{n:} \rightarrow \mathrm{C}^{k}$ be the projection defined by

$$
\pi_{i_{1} \cdots i_{k}}\left(z_{1}, \cdots, z_{n}\right)=\left(z_{i_{1}}, \cdots, z_{i_{k}}\right) .
$$

For $s=\left(z_{i_{1}}^{0}, \cdots, z_{i_{k}}^{0}\right) \in \Omega_{i_{1}} \times \cdots \times \Omega_{i_{k}}$, denote the sheaf

$$
\left(\mathcal{F} / \sum_{\mu=1}^{k}\left(z_{i_{\mu}}-z_{i_{\mu}}^{0}\right) \mathcal{F}\right) \mid \Omega \cap \pi_{i_{1} \cdots i_{k}}^{-1}(s)
$$

by $\mathcal{F}_{i_{1} \cdots i_{k}}(s)$, and denote the set

$$
\begin{aligned}
& \left\{\left(z_{1}, \cdots, z_{n}\right) \in \mathrm{C}^{n} \mid z_{i}=z_{i}^{0} \text { for } i=i_{1}, \cdots, i_{k}\right. \\
& \left.\qquad z_{j} \in G_{j} \text { for } j \neq i_{1}, \cdots, i_{k}\right\}
\end{aligned}
$$

by $G_{i_{1} \cdots i_{k}}(s)$.

We are going to prove the following $(\mathrm{a})_{k}$ and $(\mathrm{b})_{k}$ for $1 \leq k \leq n+1$ by descending induction on $k$.

$(a)_{k}$

$\mathcal{F}$ is $\pi_{i_{1} \cdots i_{k}}$-flat on $G^{-} \cap \pi_{i_{1} \cdots i_{k}}^{-1}\left(\partial G_{i_{1}} \times \cdots \times \partial G_{i_{k}}\right)$.

(b) $k$

$$
\text { For } s \in \partial G_{i_{1}} \times \cdots \times \partial G_{i_{k}}
$$

$$
S_{l}\left(\mathcal{F}_{i_{1} \cdots i_{k}}(s)\right) \cap \partial_{l+1} G_{i_{1} \cdots i_{k}}(s)=\not \supset \text { for } 0 \leq l<n-k \text {. }
$$

Since $(a)_{n+1}$ and $(b)_{n+1}$ are vacuous statements, it suffices to prove the following statements.

(I) $(\mathrm{a})_{k+1}$ and $(\mathrm{b})_{k+1} \Rightarrow(\mathrm{b})_{k}$.

(II) $(\mathrm{a})_{k+1}$ and $(\mathrm{b})_{k} \Rightarrow(\mathrm{a})_{k}$.

To prove (I), we can assume that $i_{\mu}=\mu$ for $1 \leq \mu \leq k$. Take $1 \leq l \leq n-k$ and take $s^{*} \in \partial_{l} G_{1 \ldots k}(s)$. We can assume w.l.o.g. that $s^{*}=\left(z_{1}^{0}, \ldots, z_{n}^{0}\right)$ with $z_{i}^{0} \in \partial G_{i}$ for $k+1 \leq i \leq k+l$. We have to show that $s^{*} \notin s_{l-1}\left(\mathscr{F}_{1 \ldots k}(s)\right)^{n}$. Suppose the contrary. Then the homological codimension of $\mathcal{F}_{1 \ldots k}(s)$ at $s^{*}$ is $\leq l-1$. Since, by $(a)_{k+1}, \mathcal{F}$ is $\pi_{1} \ldots(k+1)^{-f l a t}$ on $\partial G_{1} \times \cdots \times \partial G_{k+1} \times G_{k+2}^{-} \times \cdots \times G_{n}^{-}$, it follows that $z_{k+1}-z_{k+1}^{0}$ is not a zero-divisor for $\mathcal{F}_{1 \ldots k}(s)$ at $s^{*}$. Let $s^{\prime}=$ 
$\left(z_{1}^{0}, \ldots, z_{k+1}^{0}\right)$. Since

$$
\mathcal{F}_{1 \cdots(k+1)}\left(s^{\prime}\right)=\mathcal{F}_{1 \cdots k}(s) /\left(z_{k+1}-z_{k+1}^{0}\right) \mathcal{F}_{1 \cdots k}(s),
$$

we conclude that the homological codimension of $\mathcal{F}_{1 \ldots(k+1)}\left(s^{\prime}\right)$ at $s^{*}$ is $\leq l-2$. However, since $s^{*} \in \partial_{l-1} G_{1 \ldots(k+1)}\left(s^{\prime}\right)$, it follows from $(b)_{k+1}$ that $s^{*} \notin$ $s_{l-2}\left(\mathcal{F}_{1 \ldots(k+1)}\left(s^{\prime}\right)\right)$. We have a contradiction. (I) is proved.

To prove (II), we can again assume that $i_{\mu}=\mu$ for $1 \leq \mu \leq k$. Let $G^{\prime}=G_{1} \times$ $\cdots \times G_{k}$ and $G^{\prime \prime}=G_{k+1} \times \cdots \times G_{n}$. Let $E^{(i)}=B\left(G^{\prime \prime},{ }_{n} \mathcal{E}^{p_{i}}\right) \mid \Omega_{1} \times \cdots \times \Omega_{k}$ for $0 \leq i \leq m$. Let

$$
0 \rightarrow E^{(m)} \stackrel{\phi^{(m)}}{\longrightarrow} \ldots \stackrel{\phi^{(2)}}{\longrightarrow} E^{(1)} \stackrel{\phi^{(1)}}{\longrightarrow} E^{(0)}
$$

be induced by (*). We will apply Lemma (7.1) to (\#) to obtain (II).

In the rest of this proof, for $s \in \partial_{k} G^{\prime}, \mathcal{F}(s)$ stands for $\mathcal{F}_{1 \ldots k}(s) . \mathcal{F}(s)$ is regarded as a sheaf on $\Omega_{k+1} \times \cdots \times \Omega_{n}$. $\mathcal{G}(s)$ and ${ }_{n} \mathcal{O}^{p_{i}}(s)$ have meanings similar to $\mathcal{F}(s)$. Since $S_{l}\left(\varrho_{)} \cap \partial_{l+1} G=\varnothing\right.$ for $0 \leq l<n$, it follows from Proposition (1.4) that

$$
\text { O is } \pi_{1 \ldots k} \text {-flat on }\left(\partial_{k} G^{\prime}\right) \times\left(G^{\prime \prime}\right)^{-}
$$

and

$$
S_{l}(G(s)) \cap \partial_{l+1} G^{\prime \prime}=\varnothing \quad \text { for } 0 \leq l<n-k \text { and } s \in \partial_{k} G^{\prime}
$$

By Proposition (6.2) and ( $t \dagger)$,

$$
G^{\prime \prime} \text { is strongly } \varrho(s) \text {-privileged for } s \in \partial_{k} G^{\prime} \text {. }
$$

By (2.4) and ( $\dagger$ ), there exist Stein open subsets $U_{1}, \ldots, U_{r}$ of $\mathbf{C}^{k}$ such that

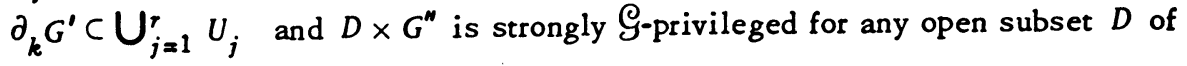
any $U_{j}$.

We claim that the complex

$$
0 \rightarrow \mathcal{O}\left(E^{(m)}\right) \stackrel{\psi^{(m)}}{\longrightarrow} \cdots \rightarrow \mathcal{O}\left(E^{(1)}\right) \stackrel{\psi^{(1)}}{\longrightarrow} \mathcal{O}\left(E^{(0)}\right)
$$

induced by (\#) is exact on $\bigcup_{j=1}^{r} U_{j}$. To verify the claim, take $1 \leq j \leq r$ and open subsets $D \subset \subset D^{\prime} \subset U_{j}$. Take $f^{\prime} \in \Gamma\left(D^{\prime}, \mathcal{C}\left(E^{(i)}\right)\right)$ for some $0<i \leq m$ such that $\psi^{(i)}\left(f^{\prime}\right)=0 . f^{\prime} \mid D$ corresponds uniquely to an element $f \in B\left(D \times G^{n},{ }_{n} \mathcal{C}^{p_{i}}\right)$ and $f$ satisfies $\alpha_{i}(f)=0$. If $i=m$, from $\operatorname{Ker} \alpha_{m}=0$ it follows that $f=0$ and $f^{\prime} \mid D=0$. If $1 \leq i<m$, since $D \times G^{\prime \prime}$ is strongly $\varrho_{\text {-privileged, the sequence }}$

$$
B\left(D \times G^{n},{ }_{n} \Theta^{p_{i}+1}\right) \rightarrow B\left(D \times G^{\prime \prime},{ }_{n} \Theta^{p i}\right) \rightarrow B\left(D \times G^{\prime \prime},{ }_{n} \Theta^{p_{i-1}}\right)
$$

induced by $(*)$ is exact (the exactness for the case $i=1$ comes from the injec- 
tivity of the natural map $\left.B\left(D \times G^{\prime \prime}, \varrho\right) \rightarrow \Gamma\left(D \times G^{\prime \prime}, \varrho\right)\right)$. Therefore there exists $g \in B\left(D \times G^{\prime \prime},{ }_{n} \mathcal{C}^{p_{i+1}}\right)$ such that $\alpha_{i+1}(g)=f \cdot g$ corresponds uniquely to an element $g^{\prime} \in \Gamma\left(D^{n}, \mathcal{Q}\left(E^{(i+1)}\right)\right)$. $\psi^{(i+1)}\left(g^{\prime}\right)=f^{\prime} \mid D$. The claim is verified.

Next we claim that $\psi_{s}^{(i)}$ is direct for $1 \leq i \leq m$ and $s \in \partial_{k} G^{\prime}$. By virtue of (**), we need only verify that $\psi_{s}^{(1)}$ is direct for $s \in \partial_{k} G^{\prime}$. Fix $s \in \partial_{k} G^{\prime}$. Since, by (a) $k+1, \mathcal{F}$ is $\pi_{1 \cdots k i}$-flat on $G^{-} \cap \pi_{1 \cdots k i}^{-1}\left(\partial G_{1} \times \cdots \times \partial G_{k} \times \partial G_{i}\right)$ for $k+1 \leq i \leq n$, it follows that

$$
\mathcal{F} \text { is } \pi_{1 \ldots k} \text {-flat on }\{s\} \times \partial G^{\prime \prime} .
$$

By $(\dagger)$ and Lemma (7.3), $\mathcal{F}(s)$ admits a finite free resolution on an open neighborhood of $\left(G^{n}\right)^{-}$in $\Omega_{k+1} \times \cdots \times \Omega_{n}$. By applying Lemma (7.4) to the exact sequence

$$
{ }_{n}^{\Theta^{p}}(s) \rightarrow{ }_{n} \Theta^{p}(s) \rightarrow \mathcal{F}(s) \rightarrow 0
$$

on $\Omega_{k+1} \times \cdots \times \Omega_{n}$, we conclude from (b) $k$ and Proposition $(6.2)$ that $\psi_{s}^{(1)}$ is direct.

Since $G$ is weakly $\mathcal{F}$-privileged, for $0<i \leq m$ the map $B\left(G^{\prime}, E^{(i)}\right) \rightarrow$ $B\left(G^{\prime}, E^{(i-1)}\right)$ induced by $\phi^{(i)}$ has a closed image. By Lemma (7.1), the sequence (\#), when restricted to any point of $\partial_{k} G^{\prime}$, is exact. It follows from Lemma (7.5) and (b) that (a) $k$ holds. (II) is proved.

The proposition follows from $(a)_{1}, \ldots,(a)_{n}$. Q.E.D.

8.

(8.1) Proposition. Suppose $\Omega$ is an open subset of $\mathbf{C}^{n}$ and $\mathcal{F}$ is a coberent analytic sheaf on $\Omega$ admitting a finite free resolution on $\Omega$. Suppose $G$ is a polydomain in $\mathbf{C}^{n}$ sucb that $G \subset C \Omega$. If $G$ is semilocally weakly $F_{\text {-privileged, }}$ then $G$ is weakly $\mathcal{F}$-privileged.

Proof. Let

$$
0 \rightarrow{ }_{n} \Theta^{p_{m}} \rightarrow \cdots \rightarrow{ }_{n} \Theta^{p_{1}} \stackrel{\phi}{\rightarrow}{ }_{n} \Theta^{p_{0}} \rightarrow \mathcal{F} \rightarrow 0
$$

be the finite free resolution of $\mathcal{F}$ on $\Omega$. We prove by induction on $m$. Let $\mathcal{S}=$ $\operatorname{Ker} \phi$. By induction hypothesis and Propositions (7.6) and (6.2), $G$ is locally strongly S-privileged. By Lemma (5.2), the sequence

$$
0 \rightarrow \Theta_{G^{-}}^{p_{m}} \rightarrow \cdots \rightarrow \Theta_{G^{-}}^{p_{1}} \stackrel{\psi}{\rightarrow} \Theta_{G^{-}}^{p_{0}}
$$

is exact on $G^{-}$. Since $B\left(G,{ }_{n} \mathcal{O}^{p_{i}}\right) \approx \Gamma\left(G^{-}, \mathcal{O}_{G_{i}}^{p_{i}}\right)$ for $i=0,1$, to finish the proof, we need only show that the map $\tilde{\psi}: \Gamma\left(G^{-}, \mathcal{O}_{G^{-}}^{p_{1}}\right) \rightarrow \Gamma\left(G^{-}, \mathcal{O}_{G^{-}}^{p_{0}}\right)$ has a closed image. 
Suppose $f \in(\operatorname{Im} \tilde{\psi})^{-}$. Since $G$ is semilocally weakly $\mathcal{F}_{\text {-privileged, there ex- }}$ ist open subsets $U_{1}, \ldots, U_{k}$ of $C^{n}$ such that $\partial G \subset \bigcup_{i=1}^{k} U_{i}$ and $G \cap U_{i}$ is weakly $\mathcal{F}_{\text {-privileged }}(1 \leq i \leq k)$. For $1 \leq i \leq k$ let $a_{i}: B\left(G \cap U_{i},{ }_{n} \mathcal{E}^{p_{1}}\right) \rightarrow$ $B\left(G \cap U_{i},{ }_{n} \mathcal{C}^{p 0}\right)$ be induced by $\phi . f \mid G \cap U_{i} \in \operatorname{Im} \alpha_{i}$ for $1 \leq i \leq k$, because $f \mid G$ $\cap U_{i} \in\left(\operatorname{Im} \alpha_{i}\right)^{-}$and $\operatorname{Im} \alpha_{i}$ is closed. It follows that $f G^{-} \cap U_{i} \in \Gamma\left(G^{-} \cap U_{i}, \operatorname{Im} \psi\right)$ for $1 \leq i \leq k$. By [4, p. 85, II.D.3], $f G \in \Gamma(G, \operatorname{Im} \psi)$. Hence $f \in \Gamma\left(G^{-}, \operatorname{Im} \psi\right)$. By Corollary (4.7) and the exactness of (*), $f \in \operatorname{Im} \widetilde{\psi}$. Q.E.D.

(8.2) Proof of Main Theorem. The Main Theorem follows from Propositions (6.2), (6.4), (7.6), and (8.1).

(8.3) Remark. The Main Theorem does not hold in its full strength if the following condition is not included in its hypotheses:

Every point of $\partial G_{i}$ is a peak point of $B\left(G_{i}\right)$ for $1 \leq i \leq n$.

A trivial counterexample is the following: Let $\Delta$ be the 1 -dimensional open unit disc and let $\Delta^{\prime}=\Delta-[0,1)$. Let $G=\Delta^{\prime} \times \Delta^{\prime}$ and $\mathcal{F}$ be the maximum sheaf ideals on $\mathrm{C}^{2}$ whose zero-set is $\{0\}$. $S_{1}(\mathcal{F}) \cap \partial_{2} G \neq \varnothing$, but $G$ is strongly $\mathcal{F}$-privileged, because $B\left(\Delta^{\prime} \times \Delta^{\prime},{ }_{2} \mathcal{O}\right) \approx B\left(\Delta \times \Delta,{ }_{2} \mathcal{Q}\right)$.

However, without $(*)$ some implications of the Main Theorem still hold, e.g. (i) $\Rightarrow$ (ii), and (i) $\Rightarrow$ (vi).

9. Finally we conclude this paper by a simple proposition concerning extending uniformly continuous holomorphic functions on a subvariety of a polydomain to the polydomain. This proposition follows easily from arguments analogous to those given in [G] and from the preceding results and techniques of this paper.

(9.1) Proposition. Suppose $G$ is a bounded polydomain in $\mathbf{C}^{n}, \Omega$ is an open neigbborbood of $G^{-}$in $\mathrm{C}^{n}$, and $\left(X, \mathcal{O}_{X}\right)$ is a subvariety of pure division $r$ in $\Omega$ endowed with the reduced complex structure. Suppose the following three conditions are satisfied.

(i) $X \cap \partial_{r+1} G=\varnothing$.

(ii) $S_{k}\left(\Theta_{X}\right) \cap \partial_{k} G=\varnothing$ for $1 \leq k<r$.

(iii) If $i_{1}, \ldots, i_{r}$ are distinct elements of $\{1, \cdots, n\}$ and $x=\left(z_{1}^{0}, \ldots, z_{n}^{0}\right)$ $\epsilon G^{-} \cap X$ such that $z_{i} \in \partial G_{i}$ for $i=i_{1}, \cdots, i_{r^{\prime}}$ then the map $\mathbf{C}^{n} \rightarrow \mathbf{C}^{r}$ defined by $\left(z_{1}, \ldots, z_{n}\right) \mapsto\left(z_{i_{1}}, \ldots, z_{i_{r}}\right)$ maps some open neigbborbood of $x$ in $X$ bibolomorpbically onto an open subset of $\mathrm{C}^{r}$.

Then $B\left(G, \Theta_{X}\right)$ is canonically topologically isomorphic to the Banach space of all uniformly continuous bolomorphic functions on $G \cap X$.

There is an analog of Proposition (9.1) for uniformly bounded holomorphic functions. 
Added in proof. According to the Autorreferat written by G. Pourcin for the Zentralblatt für Mathematik (Vol. 211 (1971), p. 395), Douady has obtained, at the same time as I, a proof for Corollary (0.7).

\section{REFERENCES}

1. A. Douady, Le problème des modules pour les sous -espaces analytiques compacts d'un espace analytique donné, Ann. Inst. Fourier (Grenoble) 16 (1966), fasc. 1, 1-95. MR 34 \#2940.

2. A. Douady, J. Frisch and A. Hirschowitz, Recouvrements privilégiés, Ann. Inst. Fourier (Grenoble) 22 (1972), fasc. 4, 59-96.

3. T. Gamelin, Uniform algebras, Prentice-Hall, Englewood Cliffs, N. J., 1969.

4. R. C. Gunning and H. Rossi, Analytic functions of several complex variables, Prentice-Hall Series in Modern Analysis, Prentice-Hall, Englewood Cliffs, N. J., 1965. MR 31 \#4927.

5. G. Pourcin, Polycylindres privilégiés, C. R. Acad Sci. Paris Sér. A 272 (1971), $795-798$.

6. Y.-T. Siu, Sheaf cohomology with bounds and bounded holomorphic functions, Proc. Amer. Math. Soc. 21 (1969), 226-229. MR 38 \#6108.

7. Y.-T. Siu and G. Trautmann, Gap-sheaves and extension of coherent analytic subsheaves, Lecture Notes in Math., vol. 172, Springer-Verlag, Berlin and New York, 1971. MR 44 \#240.

DEPARTMENT OF MATHEMATICS, YALE UNIVERSITY, NEW HAVEN, CONNECTICUT 06520 